\title{
An Intrinsic Approach to the Geodesical Connectedness of Stationary Lorentzian Manifolds
}

\author{
Fabio Giannoni ${ }^{1}$ and PaOlo Piccione ${ }^{2}$
}

\begin{abstract}
We prove a variational principle for geodesics on a Lorentzian manifold $\mathcal{M}$ admitting a timelike Killing vector field. Using this principle and standard techniques of global nonlinear analysis we establish the existence of geodesics that join two fixed points of $\mathcal{M}$, under a suitable coercivity assumption on $\mathcal{M}$. Whenever $\mathcal{M}$ is non contractible, we also get a multiplicity result for geodesics in $\mathcal{M}$ joining two fixed points.
\end{abstract}

\section{Introduction.}

In this paper we consider the problem of the existence of geodesics that join two fixed point in a Lorentzian manifold.

We will make a symmetry assumption on the metric of our Lorentzian manifold. Namely, we will assume that our spacetime possesses a 1parameter group of (local) isometries, whose infinitesimal generator is a timelike vector field. Heuristically, this amounts to say that the coefficients of our Lorentzian metric tensor are invariant by time translation (see Lemma C.1), and so our manifold is stationary with respect to a given observer field. Such a vector field is used to prove an alternative variational principle for geodesics, and this principle allows to reduce the search of geodesics to the study of the critical points of a smooth functional which is bounded from below.

The class of stationary Lorentzian manifolds is reasonably large, and it contains examples that can be considered interesting both from a physicist's and from a mathematician's point of view. Among others, we would like to recall here the Schwarzschild space-time, the Reissner-Nordström spacetime and the Kerr space-time. We refer to [9] for a detailed description of the mentioned examples and their physical interpretation.

\footnotetext{
${ }^{1}$ Partially supported by MURST

${ }^{2}$ Supported by CAPES, Processo AEX1697/96-0
} 
Before stating the main results, we recall some basic notions of the Lorentzian geometry. The books $[4,9,11,13]$ are excellent references for a complete account of the theory and for all the background material assumed in this paper. A Lorentzian manifold is a smooth, finite dimensional manifold $\mathcal{M}$, equipped with a $(0,2)$-tensor $g$ of index 1 . The bilinear form $g(z)[\cdot, \cdot]$ on $T_{z} \mathcal{M}$ will also be denoted by $\langle\cdot, \cdot\rangle$ in the rest of the article. The points of the manifold $\mathcal{M}$ will also be called events.

A vector $v \in T_{z} \mathcal{M}$ is said timelike (resp. lightlike, spacelike) if $\langle v, v\rangle$ is negative (resp. null, positive); $v$ is called causal if it is not spacelike.

A Lorentzian manifold is said to be time-oriented if there exists a continuous vector field $Y$ on $\mathcal{M}$ such that $Y(z)$ is timelike for all $z \in \mathcal{M}$. A timelike vector field defines the past and the future of a point $z$ in $\mathcal{M}$ : a causal vector $v \in T_{z} \mathcal{M}$ is said to be future pointing (resp. past pointing) if $\langle v, Y(z)\rangle$ is negative (resp. positive).

Moreover, a timelike vector $Y$ field on $\mathcal{M}$ allows to define a Riemannian metric $g_{(\mathrm{R})}$ on $\mathcal{M}$ by setting:

$$
g_{(\mathrm{R})}(x)\left[\zeta_{1}, \zeta_{2}\right]=\left\langle\zeta_{1}, \zeta_{2}\right\rangle_{(\mathrm{R})}=\left\langle\zeta_{1}, \zeta_{2}\right\rangle-2 \frac{\left\langle\zeta_{1}, Y(x)\right\rangle\left\langle\zeta_{2}, Y(x)\right\rangle}{\langle Y(x), Y(x)\rangle}
$$

for every $x \in \mathcal{M}$ and every $\zeta_{1}, \zeta_{2} \in T_{x} \mathcal{M}$ (see [1]). Notice that, for every $\zeta \in T_{z} \mathcal{M}$, it is:

$$
\langle\zeta, \zeta\rangle_{(\mathrm{R})} \geq|\langle\zeta, \zeta\rangle|
$$

A Lorentzian manifold $\mathcal{M}$ is said to be stationary if it admits a time orientation given by a Killing timelike vector field $Y$. We recall that a vector field $Y$ on $\mathcal{M}$ is a Killing vector field if the Lie derivative $L_{Y} g$ of the metric tensor $g$ is everywhere vanishing. Equivalently, $Y$ is a Killing vector field if and only if the stages of all its local flows are isometries, i.e., if the metric tensor $g$ of $\mathcal{M}$ is invariant by the flow of $Y$.

A smooth curve $z:(a, b) \longmapsto \mathcal{M}$ is a geodesic if it satisfies the differential equation:

$$
\nabla_{\dot{z}} \dot{z} \equiv 0
$$

where $\nabla$ denotes the covariant derivative relative to the Levi-Civita connection of the metric tensor $g$.

Given an absolutely continuous curve $z$ and an absolutely continuous vector field $\zeta$ along $z$, whenever there is no danger of confusion we will denote by $\nabla_{s} \zeta$ the covariant derivative of $\zeta$ along $z$, defined for almost all $s$. 
We also denote by $\nabla^{(\mathrm{R})}$ the covariant derivative of the Levi-Civita connection of the Riemannian metric tensor $g_{(\mathrm{R})}$.

The geodesics in $\mathcal{M}$ are stationary points for the action functional $f$ :

$$
f(z)=\frac{1}{2} \int_{0}^{1}\langle\dot{z}, \dot{z}\rangle \mathrm{d} s,
$$

defined on the set of all $C^{1}$-curves $z:[0,1] \longmapsto \mathcal{M}$, with $z(0)=p$ and $z(1)=q$.

It is well known that if $z$ is a geodesic in $\mathcal{M}$, then there exists a constant $E_{z}$ such that:

$$
\langle\dot{z}(s), \dot{z}(s)\rangle=E_{z}, \quad \forall s .
$$

A geodesic $z$ is said to be timelike (resp. lightlike, spacelike) if $E_{z}$ is negative (resp. null, positive).

In this paper, we will often use the following well known characterization of Killing vector fields (see [13], Proposition 9.25). If $\mathcal{X}(\mathcal{M})$ denotes the space of all $C^{1}$-vector fields on on $\mathcal{M}$, then $Y \in \mathcal{X}(\mathcal{M})$ is Killing if and only if for every pair $W_{1}, W_{2} \in \mathcal{X}(\mathcal{M})$ it is:

$$
\left\langle\nabla_{W_{1}} Y, W_{2}\right\rangle=-\left\langle\nabla_{W_{2}} Y, W_{1}\right\rangle .
$$

In particular, if $z:] a, b[\longmapsto \mathcal{M}$ is an absolutely continuous curve and $Y$ is Killing, then

$$
\left\langle\dot{z}, \nabla_{s} Y(z)\right\rangle \equiv 0 .
$$

This implies that, if $Y$ is Killing, then for every geodesic $z$ in $\mathcal{M}$ the quantity $\langle\dot{z}, Y(z)\rangle$ is constant. We express this fact by saying that $\langle\dot{z}, Y\rangle=$ constant is a natural constraint for geodesics. Our variational principle for geodesics is based on this conservation law.

For some results concerning the structure of Lorentzian manifolds admitting a Killing vector field see e.g. [16] and the references therein.

A Lorentzian manifold $\mathcal{M}$ is said to be geodesically connected if, given any two points $p, q \in \mathcal{M}$, there exists at least one geodesic $z$ in $\mathcal{M}$ with endpoints in $p$ and $q$. The geodesical connectedness for Lorentzian manifolds is a problem much more delicate than in the Riemannian case, where the Hopf-Rinow theorem gives basically a full answer to the problem. To convince the reader on this point, it suffices to point out that there exists compact Lorentzian manifolds that are not geodesically connected (see for 
instance $[5,15])$. Even the geodesic completeness does not imply the geodesical connectedness for stationary Lorentzian manifolds; a counterexample is given by the pseudosphere $S_{1}^{n}$ of index 1 (see [4]).

The main result of this paper is the proof of the geodesical connectedness for a class of stationary Lorentzian manifolds, described by intrinsic assumptions.

Before stating our main results, we need the following definition. Let $p$ and $q$ be fixed points in $\mathcal{M}$, and consider the set of $C^{1}$-curves in $\mathcal{M}$ joining $p$ and $q$ and such that $\langle\dot{z}, Y\rangle$ is constant:

$$
\mathcal{C}_{p, q}=\mathcal{C}_{p, q}(\mathcal{M})=\left\{z \in C^{1}([0,1], \mathcal{M}): z(0)=p, z(1)=q,\langle\dot{z}, Y\rangle \equiv C_{z}\right\} .
$$

Definition 1.1. Let $c$ be a real number. The set $\mathcal{C}_{p, q}$ is said to be $c$ precompact if every sequence $\left\{z_{n}\right\}_{n \in} \subset \mathcal{C}_{p, q}$ with $f\left(z_{n}\right) \leq c$ has a uniformly convergent subsequence in $\mathcal{M}$. We say that the restriction of $f$ to $\mathcal{C}_{p, q}$ is pseudo-coercive if $\mathcal{C}_{p, q}$ is $c$-precompact for all $c \geq \inf _{\mathcal{C}_{p, q}} f$.

The $c$-precompactness condition replaces the condition of completeness for Riemannian manifolds, allowing to obtain the following existence results for geodesics in $\mathcal{M}$ joining $p$ and $q$.

Theorem 1.2. Let $p$ and $q$ be two fixed events of $\mathcal{M}$. Suppose that $\mathcal{C}_{p, q}$ is non empty, and that there exists a $c>\inf _{\mathcal{C}_{p, q}} f$ such that $\mathcal{C}_{p, q}$ is c-precompact. Then, there exists at least one geodesic in $\mathcal{M}$ joining $p$ and $q$.

In particular, if the hypotheses of Theorem 1.2 are satisfied for every pair of events $(p, q)$, then $\mathcal{M}$ is geodesically connected.

A class of examples of stationary Lorentzian manifolds satisfying the hypotheses of Theorem 1.2 will be presented in Appendix A. In Appendix B we show that, if $f$ is pseudo-coercive on $\mathcal{C}_{p, q}$ for every pair of points, then $\mathcal{M}$ is globally hyperbolic. Moreover, we give an example to show that the global hyperbolicity in general does not imply the geodesical connectedness of stationary manifolds.

Observe that the assumption that $\mathcal{C}_{p, q}$ be non empty is non trivial. Indeed, it is in general not satisfied, as the following examples shows. Consider the punctured Minkowski space $\mathcal{M}=\mathbb{R}^{3+1} \backslash\{(0,0,0,0)\}$, with coordinates $\left(x_{1}, x_{2}, x_{3}, t\right)$. The vector field $Y=\frac{\partial}{\partial t}$ is timelike and Killing. Choose $p=(-1,0,0,0), q=(1,0,0,0)$. Given any $C^{1}$-curve $z:[0,1] \longmapsto \mathcal{M}$ joining $p$ and $q$, then the function $T(s)=t(z(s))$ cannot be constant, so there 
must exist $s_{1} \in[0,1]$ such that $\left.T^{\prime}\left(s_{1}\right)=\left\langle\dot{z}\left(s_{1}\right)\right), Y\left(z\left(s_{1}\right)\right)\right\rangle \neq 0$. Clearly, there must also exist $s_{2}$ with $T^{\prime}\left(s_{2}\right)=\left\langle\dot{z}\left(s_{2}\right), Y\left(z\left(s_{2}\right)\right)\right\rangle=0$. This implies that $\mathcal{C}_{p, q}$ is empty. In general, if there exists a $C^{1}$-curve $w:[0,1] \longmapsto \mathcal{M}$ with $\langle\dot{w}, Y\rangle \neq 0$ everywhere, then there exists a unique orientation preserving reparametrization $W$ of $w$ such that $\langle\dot{W}, Y\rangle$ is constant. Namely, $W=w \circ \sigma^{-1}$, where $\sigma:[0,1] \longmapsto[0,1]$ is given by:

$$
\sigma(r)=\left(\int_{0}^{1}\langle\dot{w}, Y\rangle \mathrm{d} s\right)^{-1} \int_{0}^{r}\langle\dot{w}, Y\rangle \mathrm{d} s .
$$

If $\mathcal{M}$ is non contractible, we prove the following multiplicity result for geodesics joining two given points.

We recall that a vector field $W$ on a differentiable manifold $\mathcal{M}$ is said to be complete if its flow lines are defined on the entire real line.

Theorem 1.3. Suppose that $\mathcal{C}_{p, q}$ is non empty and that $f$ is pseudo-coercive in $\mathcal{C}_{p, q}$. Then, if $Y$ is complete and $\mathcal{M}$ is non contractible there exists a sequence $\left\{z_{n}\right\}_{n \in}$ of spacelike geodesics between $p$ and $q$ in $\mathcal{M}$ such that:

$$
\lim _{n \rightarrow \infty} f\left(z_{n}\right)=+\infty
$$

We also prove a multiplicity result for timelike geodesics. We introduce some notation needed to state it.

Suppose that $Y$ is complete. Then, for every point $q \in \mathcal{M}$, we denote by $\gamma_{q}: \mathbb{R} \longmapsto \mathcal{M}$ the maximal integral curve of $Y$ satisfying $\gamma_{q}(0)=q$. The curve $\gamma_{q}(t)$ is interpreted as the worldline of an observer through the event $q$.

Theorem 1.4. Suppose that $\mathcal{M}$ is non contractible and that $Y$ is complete. Assume the existence of $c_{0}<0$ and $t_{0}>0$ such that $|t| \geq t_{0}$ implies that $\mathcal{C}_{p, \gamma_{q}(t)} \neq \emptyset$ and it is co-precompact. If $n\left[p, \gamma_{q}(t)\right]$ denotes the number of timelike geodesics joining $p$ and $\gamma_{q}(t)$, it is:

$$
\lim _{|t| \rightarrow+\infty} n\left[p, \gamma_{q}(t)\right]=+\infty .
$$

From Theorem 1.4 and Remark 6.2 it follows easily that, under the hypotheses of Theorem 1.4 it is:

$$
\lim _{\left|t_{2}-t_{1}\right| \rightarrow+\infty} n\left[\gamma_{p}\left(t_{1}\right), \gamma_{q}\left(t_{2}\right)\right]=+\infty
$$


Theorem 1.2, Theorem 1.3 and Theorem 1.4 extend the non intrinsic results obtained in [6] and [8], where it was assumed that $\mathcal{M}$ had a global space-time splitting. Our intrinsic coercivity condition is more general than the one used in the above mentioned papers (see Appendix A).

We would also like to point out that, to avoid lengthy computations, in this paper we have omitted to treat the case of a subset with convex boundary, as it was done in [8]. Nevertheless, also this case can be treated using our intrinsic estimates and the penalization argument of [8], and it is not too difficult to prove that the results of Theorems 1.2, 1.3 and 1.4 remain true if one replaces $\mathcal{M}$ with an subset of $\mathcal{M}$ with smooth convex boundary.

The paper is organized as follows. In Section 2 we introduce our analytical framework and in Section 3 we prove a variational principle for geodesics in stationary manifolds, with the introduction of the Hilbert manifold $\mathcal{N}_{p, q}$, which is the completion of $\mathcal{C}_{p, q}$ with respect to the $H^{1,2}$-norm.

In Section 4 we show the boundedness properties of the functional $J$, which is the restriction of the action functional $f$ to $\mathcal{N}_{p, q}$, whose critical points are geodesics in $\mathcal{M}$. In Section 5 we prove the Palais-Smale condition for $J$, and using classical techniques from Critical Point Theory we derive the proof of Theorems 1.2 and 1.3. The problem of the multiplicity of timelike geodesics is studied in Section 6, where we prove Theorem 1.4. Finally, we present three Appendices to the work. In Appendix A we give some examples of manifolds that satisfy the assumptions of the theorems proven in the paper. In Appendix B we discuss the relations between the pseudo-coercivity of $f$ and the property of global hyperbolicity for $\mathcal{M}$. An example is given to prove that the global hyperbolicity does not yield the geodesical connectedness for stationary Lorentzian manifolds. This was the motivation for strengthening the property of global hyperbolicity with our c-precompactness condition. In Appendix C, for the sake of completeness, we collect a few elementary facts about the local structure of stationary Lorentzian manifolds that were used in the course of the paper.

Acknowledgment. The authors are pleased to acknowledge the help provided by Miguel Sánchez, who suggested the counterexample presented in Appendix B. 


\section{The Functional Framework.}

We use the auxiliary Riemannian metric (1.1) to define the main spaces of our functional framework. We denote by $\operatorname{dist}(\cdot, \cdot)$ the distance function on $\mathcal{M} \times \mathcal{M}$ induced by $g_{(\mathrm{R})}$. We assume that $Y$ is a smooth timelike vector field defined in $\mathcal{M}$.

Let $p$ and $q$ two arbitrarily fixed points in $\mathcal{M}$. We will denote by $\Omega_{p, q}^{1,2}(\mathcal{M})$ the space of $H^{1,2}$-curves in $\mathcal{M}$ joining $p$ and $q$ :

$$
\begin{aligned}
\Omega_{p, q}^{1,2}=\Omega_{p, q}^{1,2}(\mathcal{M})=\{z:[0,1] & \longmapsto \mathcal{M} \mid z \text { absolutely continuous, } \\
& \left.z(0)=p \xi(1)=q, \int_{0}^{1}\langle\dot{z}, \dot{z}\rangle_{(\mathrm{R})} \mathrm{d} s<+\infty\right\} .
\end{aligned}
$$

It is well known that $\Omega_{p, q}^{1,2}(\mathcal{M})$ is an infinite dimensional Hilbert manifold (see [14]); for $z \in \Omega_{p, q}^{1,2}(\mathcal{M})$ the tangent space $T_{z} \Omega_{p, q}^{1,2}$ may be identified with the space of $H_{0}^{1,2}$-vector fields along $z$ :

$$
T_{z} \Omega_{p, q}^{1,2}(\mathcal{M})=\left\{\zeta \in H^{1,2}([0,1], T \mathcal{M}), \zeta(0)=\zeta(1)=0, \zeta(s) \in T_{z(s)} \mathcal{M} \forall s\right\},
$$

where $H^{1,2}([0,1], T \mathcal{M})$ is defined as:

$$
\begin{aligned}
H^{1,2}([0,1], T \mathcal{M})=\{\zeta:[0,1] \longmapsto T \mathcal{M}: \zeta \text { absolutely continuous, } \\
\left.\qquad \int_{0}^{1}\left\langle\nabla_{\dot{z}}^{(\mathrm{R})} \zeta, \nabla_{\dot{z}}^{(\mathrm{R})} \zeta\right\rangle_{(\mathrm{R})} \mathrm{d} s<+\infty\right\} .
\end{aligned}
$$

Observe that $T_{z} \Omega_{p, q}^{1,2}$ is a Hilbert space with respect to the norm:

$$
\|\zeta\|_{*}=\left(\int_{0}^{1}\left\langle\nabla_{s}^{(\mathrm{R})} \zeta, \nabla_{s}^{(\mathrm{R})} \zeta\right\rangle_{(\mathrm{R})} \mathrm{d} s\right)^{\frac{1}{2}} .
$$

For $r \geq 1$, we will denote by $L^{r}([0,1], T \mathcal{M})$ the set of all $r$-integrable vector valued functions on $[0,1]$ with values in $T \mathcal{M}$ :

$$
\begin{aligned}
& L^{r}([0,1], T \mathcal{M})=\{\zeta:[0,1] \longmapsto \\
& \qquad \mathcal{M} \text { measurable : } \\
&\left.\|\zeta\|_{r} \equiv\left(\int_{0}^{1}\langle\zeta(s), \zeta(s)\rangle_{(\mathrm{R})}^{\frac{r}{2}} \mathrm{~d} s\right)^{\frac{1}{r}}<+\infty\right\} .
\end{aligned}
$$


Similarly, one defines the space $L^{\infty}([0,1], T \mathcal{M})$ as the set of all measurable $\operatorname{maps} \zeta:[0,1] \longmapsto T \mathcal{M}$ for which

$$
\|\zeta\|_{\infty}=\operatorname{ess} \sup \sqrt{\langle\zeta(s), \zeta(s)\rangle_{(\mathrm{R})}}<+\infty .
$$

We say that a sequence $\zeta_{n}$ in $L^{r}([0,1], \mathcal{M})$ tends to 0 if $\left\|\zeta_{n}\right\|_{r}$ converges to 0 as $n$ goes to infinity.

The action functional $f$ on $\Omega_{p, q}^{1,2}(\mathcal{M})$ is defined by:

$$
f(z)=\frac{1}{2} \int_{0}^{1}\langle\dot{z}, \dot{z}\rangle \mathrm{d} s
$$

observe that, by (1.2), it is $|\langle\dot{z}, \dot{z}\rangle| \leq\langle\dot{z}, \dot{z}\rangle_{(\mathrm{R})}$, hence the integral in (2.2) makes sense for $z \in \Omega_{p, q}^{1,2}(\mathcal{M})$. The action functional is smooth on $\Omega_{p, q}^{1,2}(\mathcal{M})$, and its differential is given by:

$$
f^{\prime}(z)[\zeta]=\int_{0}^{1}\left\langle\dot{z}, \nabla_{s} \zeta\right\rangle \mathrm{d} s
$$

for every $\zeta \in T_{z} \Omega_{p, q}^{1,2}(\mathcal{M})$. Its critical points are smooth curves that satisfy the equation (1.3), hence they are geodesics. $\Omega_{p, q}^{1,2}$ by:

We write $f$ as the sum $f=f_{1}+f_{2}$ of two smooth functionals defined on

$$
f_{1}(z)=\frac{1}{2} \int_{0}^{1}\left(\langle\dot{z}, \dot{z}\rangle-\frac{\langle\dot{z}, Y\rangle^{2}}{\langle Y, Y\rangle}\right) \mathrm{d} s, \quad f_{2}(z)=\frac{1}{2} \int_{0}^{1} \frac{\langle\dot{z}, Y\rangle^{2}}{\langle Y, Y\rangle} \mathrm{d} s .
$$

Using the wrong way Schwartz's inequality, it is easy to see that $f_{1}(z) \geq 0$ for all $z \in \Omega_{p, q}^{1,2}$. Observe also that $f_{1}$ is the energy functional relative to a sub-Riemannian structure defined on $Y^{\perp}$, the orthogonal distribution to the vector field $Y$.

We denote by $\mathcal{W}$ the distribution on the manifold $\Omega_{p, q}^{1,2}(\mathcal{M})$ consisting of vector fields parallel to the timelike vector field $Y$ :

$$
\mathcal{W}=\left\{(z, \zeta) \in T \Omega_{p, q}^{1,2}(\mathcal{M}) \mid \zeta(s) \| Y(z(s)) \forall s \in[0,1]\right\} .
$$

Since $Y$ is smooth, it follows immediately that $\mathcal{W}$ is a smooth distribution on $\Omega_{p, q}^{1,2}(\mathcal{M})$. We set $\Pi(z, \zeta)=z$ the projection of $\mathcal{W}$ onto $\Omega_{p, q}^{1,2}(\mathcal{M})$, and for $z \in \Omega_{p, q}^{1,2}(\mathcal{M}), \mathcal{W}_{z}$ will denote the subspace of $T_{z} \Omega_{p, q}^{1,2}(\mathcal{M})$ given by $\Pi^{-1}(z)$.

We will denote by $H_{0}^{1,2}([0,1], \mathbb{R})$ the Hilbert space of of all functions $\mu:[0,1] \longmapsto \mathbb{R}$ of class $H^{1,2}$ such that $\mu(0)=\mu(1)=0$. Observe that 
the pair $(z, \zeta) \in T \Omega_{p, q}^{1,2}$ belongs to $\mathcal{W}$ if and only if $\zeta=\mu \cdot Y(z)$ for some $\mu \in H_{0}^{1,2}([0,1], \mathbb{R})$.

Finally, we introduce the space $\mathcal{N}_{p, q}(\mathcal{M})$ of curves $z$ in $\Omega_{p, q}^{1,2}(\mathcal{M})$ such that the the inner product $\langle\dot{z}, Y\rangle$ is constant:

$\mathcal{N}_{p, q}=\mathcal{N}_{p, q}(\mathcal{M})=\left\{z \in \Omega_{p, q}^{1,2}(\mathcal{M}) \mid\langle\dot{z}(s), Y(z(s))\rangle\right.$ is constant a.e. on $\left.[0,1]\right\}$.

Observe that the curves in $\mathcal{N}_{p, q}$ have less regularity of the curves in $\mathcal{C}_{p, q}$. Using standard arguments in Sobolev spaces, one sees that the set $\mathcal{C}_{p, q}$ is contained as a dense subset of $\mathcal{N}_{p, q}$. Thus, in the statements of Definition 1.1 and Theorems 1.2, 1.3, and 1.4 we can replace the space $\mathcal{C}_{p, q}$ with $\mathcal{N}_{p, q}$. The reason for introducing the space $\mathcal{N}_{p, q}$ is that it is the natural space for obtaining the Palais-Smale compactness condition for the action functional. The details of this fact will be discussed in section 5 .

If $Y$ is a Killing vector field, then $\mathcal{N}_{p, q}$ can be described as the set of curves $z \in \Omega_{p, q}^{1,2}$ such that the derivative $f^{\prime}(z)$ vanishes in the directions of $\mathcal{W}:$

Proposition 2.1. Suppose that $Y$ is Killing. Then it is

$$
\mathcal{N}_{p, q}=\left\{z \in \Omega_{p, q}^{1,2}(\mathcal{M}) \mid f^{\prime}(z)[\zeta]=0, \quad \forall \zeta \in \mathcal{W}_{z}\right\}
$$

Proof. Let $(z, \zeta)$ be an element of $\mathcal{W}$, with $\zeta(s)=\mu(s) \cdot Y(z(s))$ for some arbitrary $\mu \in H_{0}^{1,2}([0,1], \mathbb{R})$. Since $Y$ is Killing, then $\left\langle\dot{z}, \nabla_{s} Y\right\rangle$ vanishes identically on $[0,1]$, hence

$$
\begin{aligned}
f^{\prime}(z)[\zeta] & =\int_{0}^{1}\left\langle\dot{z}, \nabla_{s}(\mu Y(z)\rangle \mathrm{d} s=\right. \\
& =\int_{0}^{1}\left(\mu\left\langle\dot{z}, \nabla_{s} Y(z)\right\rangle+\mu^{\prime}\langle\dot{z}, Y(z)\rangle\right) \mathrm{d} s= \\
& =\int_{0}^{1} \mu^{\prime}\langle\dot{z}, Y(z)\rangle \mathrm{d} s .
\end{aligned}
$$

The latter integral in $(2.8)$ is null for every $\mu \in H_{0}^{1,2}([0,1], \mathbb{R})$ if and only if $\langle\dot{z}, Y(z)\rangle$ is constant a.e., and we are done.

We conclude the Section by proving a complementary result that shows that the space $\mathcal{N}_{p, q}$ can be described only in terms of the functional $f_{2}$. 
The meaning of the following Lemma is that the functional $f_{1}$, which is the energy functional associated to a sub-Riemannian structure on $\mathcal{M}$, is really the spatial part of the action functional $f$.

Lemma 2.2. If $(z, \zeta) \in \mathcal{W}$, then $f_{1}^{\prime}(z)[\zeta]=0$.

Proof. Consider the functional $g(z)=\frac{1}{2} \int_{0}^{1} \frac{\langle\dot{z}, Y\rangle^{2}}{\langle Y, Y\rangle}$ ds. Let $\mu \in H_{0}^{1,2}([0,1], \mathbb{R})$ and $\zeta=\mu \cdot Y$. Since $Y$ is Killing, we have:

$$
\left\langle\nabla_{\zeta} Y, Y\right\rangle=\mu\left\langle\nabla_{Y} Y, Y\right\rangle=0
$$

and

$$
\left\langle\nabla_{\dot{z}} \zeta, Y\right\rangle=\mu^{\prime}\langle Y, Y\rangle-\left\langle\dot{z}, \nabla_{\zeta} Y\right\rangle
$$

We compute the Gateaux derivative $g^{\prime}(z)[\zeta]$ as follows:

$$
\begin{aligned}
& g^{\prime}(z)[\mu Y]=\int_{0}^{1} \frac{\langle\dot{z}, Y\rangle\langle Y, Y\rangle\left[\left\langle\nabla_{\dot{z}} \zeta, Y\right\rangle+\left\langle\dot{z}, \nabla_{\zeta} Y\right\rangle\right]-\langle\dot{z}, Y\rangle^{2}\left\langle\nabla_{\zeta} Y, Y\right\rangle}{\langle Y, Y\rangle^{2}} \mathrm{~d} s= \\
& (2.9) \quad=\int_{0}^{1} \mu^{\prime}\langle\dot{z}, Y\rangle, \mathrm{d} s .
\end{aligned}
$$

Considering that $f_{1}(z)=f(z)-g(z)$, from (2.8) and (2.9) we obtain the thesis.

From (2.7) and Lemma 2.2 we obtain immediately the following characterization of the space $\mathcal{N}_{p, q}$ :

Corollary 2.3. $\mathcal{N}_{p, q}=\left\{z \in \Omega_{p, q}^{1,2}: f_{2}^{\prime}(z)[\zeta]=0, \forall \zeta \in \mathcal{W}_{z}\right\}$.

The restriction of the action functional $f$ on $\mathcal{N}_{p, q}$ will be denoted by $J$ :

$$
J=\left.f\right|_{\mathcal{N}_{p, q}} .
$$

For $c \in \mathbb{R}$, we denote by $J^{c}$ the $c$-sublevel of the functional $J$ in $\mathcal{N}_{p, q}$ :

$$
J^{c}=\left\{z \in \mathcal{N}_{p, q}: J(z) \leq c\right\} .
$$

We will show in section 3 that the set $\mathcal{N}_{p, q}$ has the structure of an infinite dimensional $C^{2}$-manifold, and that the set of critical points of $J$ in $\mathcal{N}_{p, q}$ coincides with the set of critical points of $f$ in $\Omega_{p, q}^{1,2}$. Moreover, in 
section 4 we prove that, under the hypothesis of Theorem 1.2, the functional $J$ is bounded from below on $\mathcal{N}_{p, q}$.

\section{A Variational Principle for Geodesics.}

With the aim of proving a variational principle for geodesics, we need to show the regularity of the manifold $\mathcal{N}_{p, q}$. This is done in the next:

Proposition 3.1. The set $\mathcal{N}_{p, q}$ is a $C^{2}$-submanifold of $\Omega_{p, q}^{1,2}$.

Proof. By definition, it is

$$
\mathcal{N}_{p, q}=\left\{z \in \Omega_{p, q}^{1,2}:\langle\dot{z}, Y(z)\rangle \text { is constant a.e. on }[0,1]\right\} .
$$

and so we can write $\mathcal{N}_{p, q}$ as the inverse image:

$$
\mathcal{N}_{p, q}=F^{-1}(\mathcal{C})
$$

where

$$
\begin{aligned}
F: \Omega_{p, q}^{1,2} & \longmapsto L^{2}([0,1], \mathbb{R}) \\
z & \longmapsto\langle\dot{z}, Y(z)\rangle,
\end{aligned}
$$

and $\mathcal{C}$ is the regular submanifold of $L^{2}([0,1], \mathbb{R})$ consisting of all functions which are constant almost everywhere on $[0,1]$.

The map $F$ of (3.1) is of class $C^{2}$, and its Gateaux derivative is easily computed as follows:

$$
F^{\prime}(z)[\zeta]=\left\langle\nabla_{s} \zeta, Y(z)\right\rangle+\left\langle\dot{z}, \nabla_{\zeta} Y(z)\right\rangle
$$

where $z \in \Omega_{p, q}^{1,2}, \zeta \in T_{z} \Omega_{p, q}^{1,2}$ and $\nabla_{\zeta} Y(z)$ is the covariant derivative of $Y$ along the vector field $\zeta$.

By a generalization of the Implicit Function Theorem (see Proposition 3.II.2 of [10]), in order to prove that $\mathcal{N}_{p, q}$ is a regular submanifold of $\Omega_{p, q}^{1,2}$ it suffices to show that the composite map:

$$
T_{z} \Omega_{p, q}^{1,2} \stackrel{F^{\prime}(z)}{\longrightarrow} T_{F(z)} L^{2}([0,1], \mathbb{R}) \stackrel{\pi}{\longrightarrow} T_{F(z)} L^{2}([0,1], \mathbb{R}) / T_{F(z)} \mathcal{C}
$$


is surjective ${ }^{3}$, where $\pi$ is the quotient map. This amounts to say that, for every $z \in \mathcal{N}_{p, q}$ and $h \in L^{2}([0,1], \mathbb{R})$ the equation in $\zeta$ :

$$
F^{\prime}(z)[\zeta]=h+\text { const. }
$$

can be solved in $T_{z} \Omega_{p, q}^{1,2}$. In order to prove this, we fix $z \in \mathcal{N}_{p, q}, h \in$ $L^{2}([0,1], \mathbb{R})$, and we consider the equation (3.4). We set

$$
\zeta(s)=\mu(s) \cdot Y(z(s))
$$

for some $\mu \in H^{1,2}([0,1], \mathbb{R})$, with $\mu(0)=\mu(1)=0$, so that $\zeta \in T_{z} \Omega_{p, q}^{1,2}$. Substituting $\zeta$ in (3.4), and considering that, since $Y$ is Killing, it is:

$$
\begin{aligned}
\left\langle\dot{z}, \nabla_{\zeta} Y(z)\right\rangle & =\mu \cdot\left\langle\dot{z}, \nabla_{Y} Y(z)\right\rangle= \\
& =-\mu \cdot\left\langle Y(z), \nabla_{s} Y(z)\right\rangle,
\end{aligned}
$$

we obtain the equation:

$$
\begin{aligned}
\left\langle\nabla_{s} \zeta, Y(z)\right\rangle+\left\langle\dot{z}, \nabla_{\zeta} Y(z)\right\rangle= & \mu^{\prime} \cdot\langle Y(z), Y(z)\rangle+ \\
& +\mu \cdot\left\langle\nabla_{s} Y(z), Y(z)\right\rangle-\mu \cdot\left\langle\nabla_{s} Y(z), Y(z)\right\rangle= \\
& =\mu^{\prime} \cdot\langle Y(z), Y(z)\rangle=h+C,
\end{aligned}
$$

where $C$ is a constant. Since $\langle Y, Y\rangle<0$, we can always solve (3.6) for $\mu$ by setting:

$$
\mu(s)=\int_{0}^{s} \frac{h(r)+C}{\langle Y(z(s)), Y(z(s))\rangle} \mathrm{d} r
$$

and clearly $\mu(0)=0$. Moreover, choosing

$$
C=-\int_{0}^{1} \frac{h(s)}{\langle Y(z(s)), Y(z(s))\rangle} \mathrm{d} r
$$

we also have $\mu(1)=0$, and we are done.

Using again the Implicit Function Theorem, we can easily characterize the tangent space $T_{z} \mathcal{N}_{p, q}$ :

Corollary 3.2. For $z \in \mathcal{N}_{p, q}$, the tangent space $T_{z} \mathcal{N}_{p, q}$ is identified with the set:

$T_{z} \mathcal{N}_{p, q}=\left\{\zeta \in T_{z} \Omega_{p, q}^{1,2}:\left\langle\nabla_{s} \zeta, Y(z)\right\rangle+\left\langle\dot{z}, \nabla_{\zeta} Y(z)\right\rangle\right.$ is constant a.e. on $\left.[0,1]\right\}$.

\footnotetext{
${ }^{3}$ observe that, in the case of a Hilbertian manifold, the splitting condition on the Kernel of the differential of $F$ is always satisfied.
} 
Proof. By the Implicit Function Theorem, $T_{z} \mathcal{N}_{p, q}$ is identified with the set of all $\zeta$ such that $F^{\prime}(z)[\zeta] \in T_{F(z)} \mathcal{C}$. Then, the thesis follows easily from (3.2) and the fact that $T_{F(z)} \mathcal{C}$ is identified with the set of constant functions on $[0,1]$.

Observe that, by (3.5), we also have:

$T_{z} \mathcal{N}_{p, q}=\left\{\zeta \in T_{z} \Omega_{p, q}^{1,2}:\left\langle\nabla_{s} \zeta, Y(z)\right\rangle-\left\langle\zeta, \nabla_{s} Y(z)\right\rangle\right.$ is constant a.e. on $\left.[0,1]\right\}$.

As a corollary to Theorem 3.1, we get the following variational principle for geodesics on stationary Lorentzian manifolds:

Theorem 3.3. A curve $z \in \Omega_{p, q}^{1,2}$ is a geodesic in $\mathcal{M}$ if and only if $z \in \mathcal{N}_{p, q}$ and $z$ is a critical point for the functional $J$.

Proof. If $z$ is a geodesic in $\Omega_{p, q}^{1,2}$, then $\langle\dot{z}, Y\rangle$ is constant and $z \in \mathcal{N}_{p, q}$.

Conversely, if $z \in \mathcal{N}_{p, q}$ is a critical point for the functional $J$, then $f^{\prime}(z)$ vanishes on all vectors $\zeta \in T_{z} \Omega_{p, q}^{1,2}$ of the form $\zeta=\mu \cdot Y$, with $\mu \in$ $H_{0}^{1,2}([0,1], \mathbb{R})$, and on all vectors $\zeta \in T_{z} \mathcal{N}_{p, q}$. Hence, to obtain the thesis, it suffices to show that the spaces $\left\{\mu \cdot Y: \mu \in H_{0}^{1,2}\right\}$ and $T_{z} \mathcal{N}_{p, q}$ span the entire $T_{z} \Omega_{p, q}^{1,2}$, i.e., that every $\zeta \in T_{z} \Omega_{p, q}^{1,2}$ can be written as:

$$
\zeta=\mu \cdot Y(z)+\tilde{\zeta}
$$

where $\tilde{\zeta}$ is in $T_{z} \mathcal{N}_{p, q}$ and $\mu \in H_{0}^{1,2}([0,1], \mathbb{R})$. To prove this, we consider an arbitrary $\zeta \in T_{z} \Omega_{p, q}^{1,2}$ and we search a function $\mu \in H_{0}^{1,2}$ such that the vector field:

$$
\tilde{\zeta}=\zeta-\mu \cdot Y(z)
$$

belongs to $T_{z} \mathcal{N}_{p, q}$. By Corollary 3.2, since $Y$ is Killing, this means that $\mu$ has to satisfy the differential equation:

$$
-\mu^{\prime} \cdot\langle Y(z), Y(z)\rangle+\left\langle\nabla_{s} \zeta, Y(z)\right\rangle-\left\langle\zeta, \nabla_{s} Y(z)\right\rangle=C,
$$

where $C$ is constant. Since $\langle Y, Y\rangle<0$, we can solve explicitly (3.8) by setting:

$$
\mu(s)=\int_{0}^{s} \frac{\left\langle\nabla_{s} \zeta, Y(z)\right\rangle-\left\langle\zeta, \nabla_{s} Y(z)\right\rangle-C}{\langle Y(z), Y(z)\rangle} \mathrm{d} r
$$


Clearly, $\mu(0)=0$. Moreover, setting:

$$
C=\left(\int_{0}^{1} \frac{\left\langle\nabla_{s} \zeta, Y(z)\right\rangle-\left\langle\zeta, \nabla_{s} Y(z)\right\rangle}{\langle Y(z), Y(z)\rangle} \mathrm{d} r\right)\left(\int_{0}^{1} \frac{\mathrm{d} s}{\langle Y(z), Y(z)\rangle}\right)^{-1}
$$

we also have $\mu(1)=0$ and the proof is concluded.

\section{The Lower Boundedness Condition for J.}

The aim of this section is to prove that, if $\mathcal{N}_{p, q}$ is $c$-precompact for some $c>\inf _{\mathcal{N}_{p, q}} J$, then the functional $J$ is bounded from below.

For $z \in \mathcal{N}_{p, q}$, we denote by $C_{z}$ the constant:

$$
C_{z}=\langle\dot{z}, Y\rangle
$$

We start with a basic Lemma:

Lemma 4.1. Let $c$ be any real number. If $\mathcal{N}_{p, q}$ is c-precompact, then there exists a positive constant $D$ such that $\left|C_{z}\right| \leq D$ for all $z \in J^{c}$.

Proof. Let $\left\{z_{n}\right\}_{n \in} \subset J^{c}$ be a maximizing sequence for the quantity $\left|C_{z}\right|$, i.e.

$$
\lim _{n \rightarrow \infty}\left|C_{z_{n}}\right|=\sup _{z \in J^{c}}\left|C_{z}\right| .
$$

We want to prove that $C_{z_{n}}$ is bounded. We assume, by the $c$-precompactness, that $z_{n}$ is uniformly convergent to a curve $z \in \Omega_{p, q}^{1,2}$. In particular, the sequence $z_{n}$ is eventually contained in a compact neighborhood $\mathcal{V}$ of $z([0,1])$. Using the local structure of stationary manifolds (see Appendix C), we can choose a finite number of local charts

$$
\left(\mathcal{U}_{k}, x_{1}^{k}, x_{2}^{k}, \ldots, x_{N-1}^{k}, t^{k}\right)_{k=1}^{N}
$$

where $N=\operatorname{dim}(\mathcal{M}) \geq 2$, satisfying the following properties:

(a) the $\mathcal{U}_{k}$ 's cover $\mathcal{V}$;

(b) each coordinate system $\left(x_{1}^{k}, x_{2}^{k}, \ldots, x_{N-1}^{k}, t^{k}\right)$ is adapted to $Y$, i.e., $Y=\frac{\partial}{\partial t^{k}}$ in $\mathcal{U}_{k}$ and, setting $\mathbf{x}^{k}=\left(x_{1}^{k}, x_{2}^{k}, \ldots, x_{N-1}^{k}\right)$, the metric $g$ is 
given in these coordinates by:

$$
g\left(\mathbf{x}^{k}, t\right)[(\xi, \tau),(\xi, \tau)]=\langle\xi, \xi\rangle_{0}+2\left\langle\delta^{k}\left(\mathbf{x}^{k}\right), \xi\right\rangle_{0} \tau-\beta\left(\mathbf{x}^{k}\right) \tau^{2}
$$

where, $\beta=\langle Y, Y\rangle,\langle\cdot, \cdot\rangle_{0}$ denotes the restriction of $\langle\cdot, \cdot\rangle$ on the spacelike distribution generated by $\left(\frac{\partial}{\partial x_{1}^{k}}, \frac{\partial}{\partial x_{n}^{k}}, \ldots, \frac{\partial}{\partial x_{N-1}^{k}}\right)$;

(c) $\max _{k}\left(\sup _{\mathcal{U}_{k}}\left\langle\delta^{k}\left(\mathbf{x}^{k}\right), \delta^{k}\left(\mathbf{x}^{k}\right\rangle_{0}\right)=D_{0}<+\infty\right.$;

(d) there exists a finite sequence $0=a_{0}<a_{1}<\cdots<a_{n_{0}}=1$ such that eventually $z_{n}\left(\left[a_{k-1}, a_{k}\right]\right) \subset \mathcal{U}_{k}$ for all $k=1,2, \ldots, n_{0}$ (because $z_{n}$ is uniformly convergent).

Moreover, we denote by $\nu$ and $\mu$ two positive constants such that:

$$
0<\nu \leq-\langle Y, Y\rangle \leq \mu<+\infty, \quad \text { on } \mathcal{V}
$$

and we set:

$$
\Delta_{k}=\sup _{m_{1}, m_{2} \in \mathcal{U}_{k}}\left|t^{k}\left(m_{1}\right)-t^{k}\left(m_{2}\right)\right|, \quad \Delta=\max _{k}\left(\Delta_{k}\right) .
$$

We use these coordinates and, for $n$ sufficiently large and $s \in\left[a_{k-1}, a_{k}\right]$, we write $z_{n}(s)=\left(\mathbf{x}_{n}^{k}(s), t_{n}^{k}(s)\right)$ and $Y=(\mathbf{0}, 1)$. Since $\left\langle\dot{z}_{n}, Y\right\rangle$ is constant, for $s \in\left[a_{k-1}, a_{k}\right]$ we have:

$$
C_{z_{n}}=\left\langle\dot{z}_{n}, Y\right\rangle=\left\langle\left(\dot{\mathbf{x}}_{n}^{k}, \dot{t}_{n}^{k}\right),(\mathbf{0}, 1)\right\rangle=\left\langle\delta^{k}, \dot{\mathbf{x}}_{n}^{k}\right\rangle_{0}-\beta \dot{t}_{n}^{k}
$$

hence:

$$
d_{n}^{k}=t_{n}^{k}\left(a_{k}\right)-t_{n}^{k}\left(a_{k-1}\right)=\int_{a_{k-1}}^{a_{k}} \dot{t}_{n}^{k} \mathrm{~d} s=\int_{a_{k-1}}^{a_{k}} \frac{\left\langle\delta^{k}, \dot{\mathbf{x}}_{n}^{k}\right\rangle_{0}-C_{z_{n}}}{\beta} \mathrm{d} s
$$

It follows that, for every $k, C_{z_{n}}$ can be written as:

$$
C_{z_{n}}=\left(\int_{a_{k-1}}^{a_{k}} \frac{\left\langle\delta^{k}, \dot{\mathbf{x}}_{n}^{k}\right\rangle_{0}}{\beta} \mathrm{d} s-d_{n}^{k}\right)\left(\int_{a_{k-1}}^{a_{k}} \frac{\mathrm{d} s}{\beta}\right)^{-1}
$$

Observe that, for every $n$ and $k$, it is:

$$
\left|d_{n}^{k}\right| \leq \Delta
$$


It follows immediately that, for every $k=1,2, \ldots, n_{0}$, we have:

$$
\left|C_{z_{n}}\right| \leq \frac{D_{0} \mu}{\nu} \int_{a_{k-1}}^{a_{k}} \sqrt{\left\langle\dot{\mathbf{x}}_{n}^{k}, \dot{\mathbf{x}}_{n}^{k}\right\rangle_{0}} \mathrm{~d} s+\frac{\Delta \mu}{a_{k}-a_{k-1}} .
$$

In order to prove the Theorem, we need to show that the integral

$$
\int_{a_{k-1}}^{a_{k}} \sqrt{\left\langle\dot{\mathbf{x}}_{n}^{k}, \dot{\mathbf{x}}_{n}^{k}\right\rangle_{0}} \mathrm{~d} s
$$

is bounded for at least one value of $k$. We will indeed show that the integral (4.6) is bounded on $n$ for every $k$.

From (4.3), we compute as follows:

$$
\begin{aligned}
\int_{a_{k-1}}^{a_{k}}\left\langle\dot{z}_{n}, \dot{z}_{n}\right\rangle \mathrm{d} s & =\int_{a_{k-1}}^{a_{k}}\left(\left\langle\dot{\mathbf{x}}_{n}^{k}, \dot{\mathbf{x}}_{n}^{k}\right\rangle_{0}+2\left\langle\delta^{k}, \dot{\mathbf{x}}_{n}^{k}\right\rangle_{0} \dot{t}_{n}^{k}-\beta \cdot\left(\dot{t}_{n}^{k}\right)^{2}\right) \mathrm{d} s= \\
& =\int_{a_{k-1}}^{a_{k}}\left(\left\langle\dot{\mathbf{x}}_{n}^{k}, \dot{\mathbf{x}}_{n}^{k}\right\rangle_{0}+\frac{\left\langle\delta^{k}, \dot{\mathbf{x}}_{n}^{k}\right\rangle_{0}^{2}}{\beta}-\frac{C_{z_{n}}^{2}}{\beta}\right) \mathrm{d} s .
\end{aligned}
$$

Substituting (4.4) in (4.7), we obtain:

$$
\begin{aligned}
\int_{a_{k-1}}^{a_{k}}\left\langle\dot{z}_{n}, \dot{z}_{n}\right\rangle \mathrm{d} s= & \int_{a_{k-1}}^{a_{k}}\left\langle\dot{\mathbf{x}}_{n}^{k}, \dot{\mathrm{x}}_{n}^{k}\right\rangle_{0} \mathrm{~d} s+\int_{a_{k-1}}^{a_{k}} \frac{\left\langle\delta^{k}, \dot{\mathbf{x}}_{n}^{k}\right\rangle_{0}^{2}}{\beta} \mathrm{d} s+ \\
& -\left(\int_{a_{k-1}}^{a_{k}} \frac{\mathrm{d} s}{\beta}\right)^{-1}\left(\int_{a_{k-1}}^{a_{k}} \frac{\left\langle\delta^{k}, \dot{\mathbf{x}}_{n}^{k}\right\rangle_{0}}{\beta} \mathrm{d} s\right)^{2}+ \\
& +2 d_{n}^{k}\left(\int_{a_{k-1}}^{a_{k}} \frac{\left\langle\delta^{k}, \dot{\mathbf{x}}_{n}^{k}\right\rangle_{0}}{\beta} \mathrm{d} s\right)\left(\int_{a_{k-1}}^{a_{k}} \frac{\mathrm{d} s}{\beta}\right)^{-1}+ \\
& -\left(d_{n}^{k}\right)^{2}\left(\int_{a_{k-1}}^{a_{k}} \frac{\mathrm{d} s}{\beta}\right)^{-1} .
\end{aligned}
$$

From Schwartz's inequality, we have:

$$
\left(\int_{a_{k-1}}^{a_{k}} \frac{\left\langle\delta^{k}, \dot{\mathbf{x}}_{n}^{k}\right\rangle_{0}}{\beta} \mathrm{d} s\right)^{2} \leq\left(\int_{a_{k-1}}^{a_{k}} \frac{\left\langle\delta^{k}, \dot{\mathbf{x}}_{n}^{k}\right\rangle_{0}^{2}}{\beta} \mathrm{d} s\right)\left(\int_{a_{k-1}}^{a_{k}} \frac{\mathrm{d} s}{\beta}\right)
$$


and substituting in (4.9) we obtain:

$$
\begin{aligned}
\int_{a_{k-1}}^{a_{k}}\left\langle\dot{z}_{n}, \dot{z}_{n}\right\rangle \mathrm{d} s \geq & \int_{a_{k-1}}^{a_{k}}\left\langle\dot{\mathbf{x}}_{n}^{k}, \dot{\mathbf{x}}_{n}^{k}\right\rangle_{0} \mathrm{~d} s+ \\
& +2 d_{n}^{k}\left(\int_{a_{k-1}}^{a_{k}} \frac{\left\langle\delta^{k}, \dot{\mathbf{x}}_{n}^{k}\right\rangle_{0}}{\beta} \mathrm{d} s\right)\left(\int_{a_{k-1}}^{a_{k}} \frac{\mathrm{d} s}{\beta}\right)^{-1}+ \\
& -\left(d_{n}^{k}\right)^{2}\left(\int_{a_{k-1}}^{a_{k}} \frac{\mathrm{d} s}{\beta}\right)^{-1}
\end{aligned}
$$

Finally, summing (4.10) over $k$, we obtain:

$c \geq \int_{0}^{1}\left\langle\dot{z}_{n}, \dot{z}_{n}\right\rangle \mathrm{d} s \geq \sum_{k=1}^{N} \int_{a_{k-1}}^{a_{k}}\left\langle\dot{\mathbf{x}}_{n}^{k}, \dot{\mathbf{x}}_{n}^{k}\right\rangle_{0} \mathrm{~d} s-E \cdot \sum_{k=1}^{N} \int_{a_{k-1}}^{a_{k}} \sqrt{\left\langle\dot{\mathbf{x}}_{n}^{k}, \dot{\mathbf{x}}_{n}^{k}\right\rangle_{0}} \mathrm{~d} s-F$,

where $E$ and $F$ are easily computed in terms of $\mu, \nu, \Delta, D_{0}$ and the $a_{k}$ 's. It follows that the integral (4.6) is bounded for all $k$, which proves the Lemma.

Remark 4.2. Let $c$ be a real number. If the manifold $\mathcal{N}_{p, q}$ is $c$-precompact then there exists a compact subset $K$ of $\mathcal{M}$ that contains the image $z([0,1])$ of every curve $z \in J^{c}$. In particular, if $\mathcal{N}_{p, q}$ is $c$-precompact, then there exist two positive constants $\nu=\nu(c)$ and $\mu=\mu(c)$ such that, for every $z \in J^{c}$ and every $s \in[0,1]$ :

$$
0<\nu \leq-\langle Y(z(s)), Y(z(s))\rangle \leq \mu .
$$

We are ready to prove the lower boundedness condition for $J$ :

Proposition 4.3. Suppose that $\mathcal{N}_{p, q}$ is c-precompact for some $c>\inf _{\mathcal{N}_{p, q}} J$. Then, the functional $J$ is bounded from below in $\mathcal{N}_{p, q}$.

Proof. Let $z_{n}$ be a minimizing sequence for $J$ in $J^{c}$. By the $c$-precompactness, the image of all the $z_{n}$ 's are contained in a compact subset of $\mathcal{M}$. By the definition of $g_{(\mathrm{R})}$, we have

$$
J\left(z_{n}\right)=\int_{0}^{1}\left\langle\dot{z}_{n}, \dot{z}_{n}\right\rangle_{(\mathrm{R})} \mathrm{d} s+2 C_{z_{n}}^{2} \int_{0}^{1} \frac{\mathrm{d} s}{\langle Y, Y\rangle} \geq-\frac{2}{\nu} C_{z_{n}}^{2} .
$$


The conclusion follows immediately from the fact that $C_{z_{n}}$ is bounded, as it was proven in Lemma 4.1 .

\section{Proof of Theorems 1.2 and 1.3.}

In this section we will prove Theorem 1.2 and Theorem 1.3, using standard arguments for functionals satisfying the Palais-Smale condition.

We recall that if $(X, h)$ is a Hilbertian manifold and $F: X \longmapsto \mathbb{R}$ is a $C^{1}$-functional on $X$, then $F$ is said to satisfy the Palais-Smale condition at the level $c \in \mathbb{R}$ if every sequence $\left\{x_{n}\right\}_{n \in} \subset X$ satisfying:

$(\mathrm{PS} 1)_{c} \lim _{n \rightarrow \infty} F\left(x_{n}\right)=c$,

(PS2) $\lim _{n \rightarrow \infty}\left\|F^{\prime}\left(x_{n}\right)\right\|=0$,

has a subsequence converging in $X$. The norm $\|\cdot\|$ used in (PS2) is the operator norm of $F^{\prime}\left(x_{n}\right)$ in the Hilbert space $T_{x_{n}} X$.

A sequence $x_{n}$ in $X$ that satisfies (PS1) $)_{c}$ and $(\mathrm{PS} 2)_{c}$ will be called a Palais-Smale sequence at level $c$ for the functional $F$.

We will use the following notation. If $z:[0,1] \longmapsto \mathcal{M}$ is an absolutely continuous curve and $\beta \in L^{1}([0,1], T \mathcal{M})$ is a vector field along $z$, then the covariant integral of $\beta$ along $z$, denoted by $B=\int_{z} \beta$, is the (unique) vector field along $z$ that satisfies the initial value problem:

$$
\nabla_{\dot{z}} B=\beta, \quad B(0)=0 .
$$

We need the following elementary result is semi-Riemannian geometry:

Lemma 5.1. Let $K$ be a compact subset of $\mathcal{M}$. Suppose that $z$ is an absolutely continuous curve in $K$, with $\dot{z} \in L^{1}([0,1], T \mathcal{M})$, and that $\beta \in L^{1}([0,1], T \mathcal{M})$ is a vector field along $z$. Then, the covariant integral $B=\int_{z} \beta$ of $\beta$ along $z$ is in $L^{\infty}([0,1], T \mathcal{M})$, and there exists constant $M=M(K)$ such that:

$$
\|B\|_{\infty} \leq\|\beta\|_{1} \cdot e^{M \cdot\|\dot{z}\|_{1}} .
$$

Proof. Since $K$ is covered by a finite number of charts, using local coordinates, we can assume that $\mathcal{M}$ is an open subset of $\mathbb{R}^{N}$. We denote by $|\cdot|$ 
the Euclidean norm. The vector field $B$ is the solution of the initial value problem:

$$
\frac{\mathrm{d}}{\mathrm{ds}} B=-\Gamma(z)[\dot{z}, B]+\beta, \quad B(0)=0,
$$

where

$$
\Gamma(z(s))[\cdot, \cdot]: T_{z(s)} \mathcal{M} \times T_{z(s)} \mathcal{M} \longmapsto T_{z(s)} \mathcal{M}
$$

is the bilinear map given by the Christoffel symbols $\left\{\Gamma_{i j}^{k}\right\}$ of the Lorentzian metric $g$.

Integrating $(5.2)$ on $[0, s]$, we obtain:

$$
B(s)=\int_{0}^{s} \beta \mathrm{d} r-\int_{0}^{s} \Gamma(z)[\dot{z}, B] \mathrm{d} r
$$

hence

$$
|B(s)| \leq \int_{0}^{1}|\beta| \mathrm{d} r+M \int_{0}^{s}|\dot{z}| \cdot|B| \mathrm{d} r
$$

where $M$ is the maximum of the norm of the operator $\Gamma$ on $K$. Applying Gronwall's Lemma to (5.3), we obtain:

$$
|B(s)| \leq \int_{0}^{1}|\beta| \mathrm{d} r \cdot e^{M \int_{0}^{1}|\dot{z}| \mathrm{d} r},
$$

which gives (5.1).

Remark 5.2. Suppose that $\left\{z_{n}\right\}$ is a sequence of absolutely continuous curves having image in a fixed compact subset of $\mathcal{M}$, and with $\left\|\dot{z}_{n}\right\|_{1}$ bounded. Suppose further that $\beta_{n}$ is a sequence of vector fields along the $z_{n}$ 's that tends to 0 in $L^{1}([0,1], T \mathcal{M})$. From Lemma 5.1 and its proof it follows that the sequence $B_{n}=\int_{z_{n}} \beta_{n}$ converges to 0 in $L^{\infty}([0,1], T \mathcal{M})$.

We are ready to prove the Palais-Smale condition for the functional $J$ :

Theorem 5.3. If $\mathcal{N}_{p, q}$ is c-precompact, then $J$ satisfies the Palais-Smale condition at every level $c^{\prime}<c$.

Proof. Let's fix $c^{\prime}<c$ and let $z_{n}$ be a Palais-Smale sequence at level $c^{\prime}$. Arguing as in Proposition 4.3, we see that $z_{n}$ has a subsequence (denoted again $z_{n}$ ) that converges weakly to some $z \in \Omega_{p, q}^{1,2}$. We now use the fact 
that $J^{\prime}\left(z_{n}\right)$ is infinitesimal to prove that the convergence is strong. Let $\zeta_{n} \in T_{z_{n}} \Omega_{p, q}^{1,2}$ be any sequence which is bounded in $H^{1,2}([0,1], T \mathcal{M})$; recalling (3.7), we set

$$
\zeta_{n}=\mu_{n} \cdot Y\left(z_{n}\right)+\tilde{\zeta}_{n}
$$

where $\mu=\mu_{n}$ is given by (3.9) and (3.10), while $\tilde{\zeta}_{n} \in T_{z_{n}} \mathcal{N}_{p, q}$. From (3.9) and (3.10) it follows easily that also $\tilde{\zeta}_{n}$ is bounded in $H^{1,2}([0,1], T \mathcal{M})$, hence:

$$
\lim _{n \rightarrow \infty} J^{\prime}\left(z_{n}\right)\left[\tilde{\zeta}_{n}\right]=\lim _{n \rightarrow \infty} \int_{0}^{1}\left\langle\dot{z}_{n}, \nabla_{\dot{z}_{n}} \tilde{\zeta}_{n}\right\rangle \mathrm{d} s=0
$$

Moreover, since $\left\langle\dot{z}_{n}, Y\right\rangle$ is constant and $Y$ is Killing it is:

$$
\int_{0}^{1}\left\langle\dot{z}_{n}, \nabla_{\dot{z}_{n}}\left(\mu_{n} \cdot Y\right)\right\rangle \mathrm{d} s=\int_{0}^{1} \mu_{n}^{\prime}\left\langle\dot{z}_{n}, Y\right\rangle \mathrm{d} s+\int_{0}^{1} \mu_{n}\left\langle\dot{z}_{n}, \nabla_{\dot{z}_{n}} Y\right\rangle \mathrm{d} s=0 .
$$

Putting together (5.4) and (5.5), we obtain:

$$
\lim _{n \rightarrow \infty} \int_{0}^{1}\left\langle\dot{z}_{n}, \nabla_{\dot{z}_{n}} \zeta_{n}\right\rangle \mathrm{d} s=0, \quad \forall \zeta_{n} \in T_{z_{n}} \Omega_{p, q}^{1,2} \text { bounded. }
$$

We isolate the following Lemma for future reference:

Lemma 5.4. In the above notations, there exists a sequence $\alpha_{n}$ in $T_{z_{n}} \Omega_{p, q}^{1,2}$ that tends to 0 in $L^{2}([0,1], T \mathcal{M})$ and such that:

$$
\int_{0}^{1}\left\langle\dot{z}_{n}, \nabla_{\dot{z}_{n}} \zeta_{n}\right\rangle \mathrm{d} s=\int_{0}^{1}\left\langle\alpha_{n}, \nabla_{\dot{z}_{n}} \zeta_{n}\right\rangle \mathrm{d} s
$$

Proof. We denote by $\Theta_{n}$ the vector field along $z_{n}$ which is the gradient $\nabla J\left(z_{n}\right)$ of the functional $J$ with respect to the Hilbertian norm $\|\cdot\|_{*}$ defined by (2.1). By definition, we have:

$$
\int_{0}^{1}\left\langle\dot{z}_{n}, \nabla_{\dot{z}_{n}} \zeta_{n}\right\rangle \mathrm{d} s=\int_{0}^{1}\left\langle\nabla_{\dot{z}_{n}}^{(\mathrm{R})} \Theta_{n}, \nabla_{\dot{z}_{n}}^{(\mathrm{R})} \zeta_{n}\right\rangle_{(\mathrm{R})} \mathrm{d} s
$$

and, by (5.6), the sequence of vector fields

$$
A_{n}=\nabla_{\dot{z}_{n}}^{(\mathrm{R})} \Theta_{n}
$$

goes to 0 in $L^{2}([0,1], T \mathcal{M})$. 
Using the Christoffel symbols of the metric tensors $g$ and $g_{(\mathrm{R})}$, we can express the Riemannian covariant covariant derivative $\nabla_{\dot{z}_{n}}^{(\mathrm{R})} \zeta_{n}$ in terms of the Lorentzian covariant derivative $\nabla_{\dot{z}_{n}} \zeta_{n}$. Then, we write

$$
\int_{0}^{1}\left\langle A_{n}, \nabla_{\dot{z}_{n}}^{(\mathrm{R})} \zeta_{n}\right\rangle_{(\mathrm{R})} \mathrm{d} s=\int_{0}^{1}\left\langle A_{n}, \nabla_{\dot{z}_{n}} \zeta_{n}+G\left(z_{n}\right)\left[\dot{z}_{n}\right]\left[\zeta_{n}\right]\right\rangle_{(\mathrm{R})} \mathrm{d} s
$$

where $G(z)\left[\zeta_{1}\right]\left[\zeta_{2}\right]$ is a bilinear functions in the variables $\zeta_{1}, \zeta_{2}$ which is continuous in the first variable $z$. Using (1.1), it is immediately checked the existence of two sequences $B_{n}$ and $b_{n}$ going to 0 in $L^{2}([0,1], T \mathcal{M})$ such that:

$$
\int_{0}^{1}\left\langle A_{n}, \nabla_{\dot{z}_{n}}^{(\mathrm{R})} \zeta_{n}\right\rangle_{(\mathrm{R})} \mathrm{d} s=\int_{0}^{1}\left(\left\langle B_{n}, \nabla_{\dot{z}_{n}} \zeta_{n}\right\rangle+\left\langle b_{n}, \zeta_{n}\right\rangle\right) \mathrm{d} s
$$

Now, it is:

$$
\int_{0}^{1}\left\langle b_{n}, \zeta_{n}\right\rangle \mathrm{d} s=-\int_{0}^{1}\left\langle\int_{z_{n}} b_{n}, \nabla_{\dot{z}_{n}} \zeta_{n}\right\rangle
$$

because $\zeta_{n}(0)=\zeta_{n}(1)=0$. By Remark 5.2 , it follows that $\int_{z_{n}} b_{n}$ tends to 0 uniformly, therefore (5.7) follows from (5.9).

Going back to the proof of Theorem 5.3, we now consider the sequence of vector fields

$$
\omega_{n}=\dot{z}_{n}-\alpha_{n}
$$

From (5.7) we get that $\omega_{n}$ is of class $C^{1}$ and that

$$
\nabla_{\dot{z}_{n}} \omega_{n}=0
$$

The next observation is that the $L^{2}$-norm $\left\|\omega_{n}\right\|_{2}$ of $\omega_{n}$ is bounded, because $\left\|\dot{z}_{n}\right\|_{2}$ is bounded and $\alpha_{n}$ tends to 0 in $L^{2}([0,1], T \mathcal{M})$. This implies, in particular, that, for some sequence $\left\{s_{n}\right\} \subset[0,1]$, the sequence $\left|\omega_{n}\left(s_{n}\right)\right|$ is bounded, say:

$$
\left|\omega_{n}\left(s_{n}\right)\right| \leq c_{0}, \quad \forall n \in \mathbb{N} .
$$

Once again, Gronwall's Lemma applied to the differential equation (5.11) and the boundedness condition (5.12) gives the existence of $\gamma_{0}>0$ such that:

$$
\left|\omega_{n}(s)\right| \leq c_{0} \cdot e^{\gamma_{0} \int_{0}^{1}\left|\dot{z}_{n}\right| \mathrm{d} r}, \forall s \in[0,1] .
$$


It follows that $\omega_{n}$ is bounded in $L^{\infty}$. From (5.10) it follows that $\dot{z}_{n}$ is bounded in $L^{2}$, and since $z_{n}(0)$ is fixed the sequence $z_{n}$ is uniformly bounded.

Writing equation (5.11) in coordinates, it becomes:

$$
\omega_{n}^{\prime}+\Gamma\left(z_{n}\right)\left[\dot{z}_{n}, \omega_{n}\right]=0,
$$

where $\Gamma$ is a continuous function in $z_{n}$ (that can be expressed using the Christoffel symbols of $g$ ), which is linear in the arguments $\dot{z}_{n}$ and $\omega_{n}$. From (5.13), we obtain that $\omega_{n}^{\prime}$ is bounded in $L^{2}$, and thus $\omega_{n}$ is bounded in $H^{1,2}$.

It follows that a subsequence of $\omega_{n}$ still denoted by $\omega_{n}$, is weakly convergent in $H^{1,2}$, and, in particular, $\omega_{n}$ is convergent in $L^{2}([0,1], T \mathcal{M})$. $\Omega_{p, q}^{1,2}$.

Therefore, there exists a subsequence of $z_{n}$ that tends to $z$ strongly in

By the $L^{2}$-convergence, a subsequence of $\left\langle\dot{z}_{n}, Y\right\rangle$ converges pointwise to $\langle\dot{z}, Y\rangle$ almost everywhere, which implies that $\langle\dot{z}, Y\rangle$ is constant a.e., so $z \in \mathcal{N}_{p, q}$ and the Theorem is proven.

We prove now the completeness of the $c$-sublevels of $J$ using the $c$ precompactness condition:

Proposition 5.5. Let $c \in \mathbb{R}$ be fixed. If $\mathcal{N}_{p, q}$ is c-precompact, then $J^{c^{\prime}}$ is a complete metric subspace of $\mathcal{N}_{p, q}$ for all $c^{\prime} \leq c$.

Proof. It suffices to consider the $c$-sublevel. Observe that, since all the curves in $J^{c}$ lie in a compact set (Proposition 4.2), we can assume that $\mathcal{M}$ is complete with respect to the Riemannian metric $g_{(\mathrm{R})}$. This implies that $\Omega_{p, q}^{1,2}$ is a complete Hilbertian manifold. Let $z_{n}$ be a Cauchy sequence in $J^{c}$. Then, $z_{n}$ converges to $z$ in $\Omega_{p, q}^{1,2}$, and, up to passing to a subsequence, we have convergence pointwise almost everywhere of $\left\langle\dot{z}_{n}, Y\right\rangle$ to $\langle\dot{z}, Y\rangle$. This implies that $\langle\dot{z}, Y\rangle$ is constant a.e. on $[0,1]$, so $z \in \mathcal{N}_{p, q}$. Finally, by the continuity of $J$, it is $J(z) \leq c$, and $J^{c}$ is complete.

The Palais-Smale condition, the completeness of the sublevels and the boundedness property of the functional $J$ yield immediately the existence of minimal points.

Proof of Theorem 1.2. It is a classical argument in Critical Point Theory. Thanks to the Palais-Smale condition and the completeness of the sublevels of the functionals $J$, if the infimum $i$ of $J$ on $\mathcal{N}_{p, q}$ weren't a critical value, then it would be possible to find a homotopy between the sublevels $J^{i-\eta}$ and 
$J^{i+\eta}$, where $\eta>0$ is sufficiently small. This is clearly impossible, because, for every $\eta>0, J^{i-\eta}=\emptyset$ while $J^{i+\eta} \neq \emptyset$. Hence, $J$ attains its minimum on $\mathcal{N}_{p, q}$. From Theorem 3.3, any minimal point for $J$ is a geodesic from $p$ to $q$, and we are done.

With the aim of proving Theorem 1.3, let's assume now that $\mathcal{M}$ is non contractible, and such that $\mathcal{N}_{p, q}$ is $c$-precompact for all values of the $c$. The proof of our multiplicity result is based on the Ljusternik-Schnirelman theory for Palais-Smale functionals. We recall that if $X$ is a topological space and $B$ any subset of $X$, the Ljusternik-Schnirelman category cat $X(B)$ of $B$ in $X$ is the minimal number (possibly infinite) of closed, contractible subsets of $X$ that cover $B$. The Ljusternik-Schnirelman category of $B$ in $X$ is a homotopical invariant, in the sense that $\operatorname{cat}_{X}(B)=\operatorname{cat}_{\mathcal{F}(X)}(\mathcal{F}(B))$ for every continuous map $\mathcal{F}: X \longmapsto \mathcal{F}(X)$ which is a homotopy equivalence.

A well known result by Fadell and Husseini (see [7]) states that, if $\mathcal{M}$ is non contractible, then the category of the space $\Omega_{p, q}^{1,2}(\mathcal{M})$ is infinite.

We prove now that, if $Y$ is a complete vector field, then there exists a smooth map $\mathcal{F}: \Omega_{p, q}^{1,2} \longmapsto \mathcal{N}_{p, q}$ which is a homotopy equivalence (see Proposition 5.9). This implies:

$$
\operatorname{cat}_{\mathcal{N}_{p, q}}\left(\mathcal{N}_{p, q}\right)=+\infty
$$

Let's assume that $\mathcal{M}$ is connected and that the vector field $Y$ is complete. The completeness of $Y$ is satisfied, for instance, if $\mathcal{M}$ is complete with respect to the Riemannian metric $g_{(\mathrm{R})}$ and if $Y$ satisfies the boundedness condition $-\langle Y, Y\rangle=\langle Y, Y\rangle_{(\mathrm{R})} \leq \mu$.

We denote by $\psi(x, t)$ the flow of the vector field $Y$, i.e., for every $x$ in $\mathcal{M}$, the curve $t \longmapsto \psi(x, t)$ is the unique integral curve of $Y$ starting at $x$ when $t=0$. Since $Y$ is complete, then $\psi$ gives a map defined for all times $t$ :

$$
\psi: \mathcal{M} \times \mathbb{R} \longmapsto \mathcal{M}
$$

Now, fix $p, q$ two arbitrary points in $\mathcal{M}$ and let $x:[0,1] \longmapsto \mathcal{M}$ be any smooth curve satisfying $x(0)=p$ and $x(1)=q$. We define a curve $w$ : $[0,1] \longmapsto \mathcal{M}$ by:

$$
w(s)=\psi(x(s), \phi(s))
$$


where $\phi:[0,1] \longmapsto \mathbb{R}$ is to be chosen in such a way that $\phi(0)=\phi(1)=0$ and such that $\langle\dot{w}(s), Y(w(s))\rangle=C$ is constant on $[0,1]$. Hence, we need:

$$
\begin{aligned}
\langle\dot{w}, Y(w)\rangle & =\left\langle\mathrm{d}_{x} \psi(x, \phi)[\dot{x}]+\mathrm{d}_{t} \psi(x, \phi) \phi^{\prime}, Y(w)\right\rangle= \\
& =\left\langle\mathrm{d}_{x} \psi(x, \phi)[\dot{x}], Y(w)\right\rangle+\phi^{\prime} \cdot\langle Y(\psi(x, \phi)), Y(w)\rangle= \\
& =C,
\end{aligned}
$$

where $\mathrm{d}_{x}$ and $\mathrm{d}_{t}$ denotes the differential operators with respect to the variables $x$ and $t$ respectively. In (5.16) we have used the fact that $\mathrm{d}_{t} \psi(x, \phi)=Y(\psi(x, \phi))$, which comes easily from the definition of flow. Moreover, using the group property $\psi(\psi(x, s), t)=\psi(x, s+t)$, one sees that $\mathrm{d}_{x} \psi[Y(x)]=Y(w)$. Since $\mathrm{d}_{x} \psi$ is an isometry, we have:

$$
\left\langle\mathrm{d}_{x} \psi[\dot{x}], Y(w)\right\rangle=\left\langle\mathrm{d}_{x} \psi[\dot{x}], \mathrm{d}_{x} \psi[Y(x)]\right\rangle=\langle\dot{x}, Y\rangle .
$$

Remark 5.6. Observe that, since $Y$ is Killing, the quantity $\langle Y, Y\rangle$ is constant along the flow lines of $Y$, hence it can be computed indifferently on $x(s)$ or on $w(s)$. This observation allows to omit the argument of $Y$ whenever there is no danger of confusion.

Putting together (5.16) and (5.17), we obtain that, in order for $w$ to belong to $\mathcal{N}_{p, q}$, the function $\phi$ must satisfy the first order differential equation:

$$
\phi^{\prime}=\frac{C-\langle\dot{x}, Y\rangle}{\langle Y, Y\rangle},
$$

and the boundary conditions $\phi(0)=\phi(1)=0$.

For every choice of the constant $C$, the initial value problem given by (5.18) and the initial condition $\phi(0)=0$ has a (unique) solution, which is defined on the entire interval $[0,1]$, because the right hand side of $(5.18)$ is sublinear in $\dot{x}$. If we set:

$$
C=\left(\int_{0}^{1} \frac{\langle\dot{x}, Y\rangle}{\langle Y, Y\rangle}\right) \cdot\left(\int_{0}^{1} \frac{\mathrm{d} s}{\langle Y, Y\rangle}\right)^{-1},
$$

an immediate calculation shows:

$$
\phi(1)=\int_{0}^{1} \phi^{\prime} \mathrm{d} s=0,
$$

and we are done.

Incidentally, we have proven the following: 
Lemma 5.7. Suppose that $Y$ is complete and that $\mathcal{M}$ is connected. Then, for every pair of points $p, q \in \mathcal{M}$, the sets $\mathcal{C}_{p, q}$ and $\mathcal{N}_{p, q}$ are non empty.

We have indeed proven a slightly stronger result:

Proposition 5.8. Under the same hypothesis of Proposition 5.7, any curve in $\Omega_{p, q}^{1,2}$ is homotopic to a curve in $\mathcal{N}_{p, q}$.

Proof. If $x$ is any $C^{1}$-curve in $\mathcal{M}$ joining $p$ and $q$, set

$$
H(\sigma, s)=\psi(x(s), \sigma \cdot \phi(s)),
$$

in the same notations of (5.15). This gives a homotopy between $x(\cdot)=$ $H(0, \cdot)$ and $w(\cdot)=H(1, \cdot) \in \mathcal{N}_{p, q}$.

In the notations above, we can define formally an operator:

$$
\mathcal{F}(x)=w,
$$

where $x \in \Omega_{p, q}^{1,2}$ and $w$ is the curve given by (5.15).

The map $\mathcal{F}$ is a homotopy equivalence between the spaces $\Omega_{p, q}^{1,2}$ and $\mathcal{N}_{p, q}$. This fact and other properties of $\mathcal{F}$ are collected in the next:

Proposition 5.9. The map $\mathcal{F}: \Omega_{p, q}^{1,2} \longmapsto \mathcal{N}_{p, q}$ is smooth, and it is a strong deformation retract. Moreover, it is:

$$
J(\mathcal{F}(x)) \geq f(x)
$$

where the equality sign holds if and only if $x \in \mathcal{N}_{p, q}$.

Proof. The smoothness of $\mathcal{F}$ follows easily from standard theorems on smooth dependence on the data for the ordinary differential equation (5.18).

The map $\mathcal{F}$ is the identity on $\mathcal{N}_{p, q}$. In fact, observe that, if $\langle\dot{x}, Y(x)\rangle=C$ is constant, then $\phi \equiv 0$ is the (unique) solution of (5.18), with $\phi(0)=0$. Then, $\mathcal{F}(x)(\cdot)=\psi(x(\cdot), 0)=x(\cdot)$.

The map $\mathcal{H}: \Omega_{p, q}^{1,2} \times[0,1] \longmapsto \Omega_{p, q}^{1,2}$ given by:

$$
\mathcal{H}(x, \sigma)=w_{\sigma}, \quad w_{\sigma}(s)=H(\sigma, s),
$$

where $H$ is defined by (5.20), gives a homotopy between the identity map on $\Omega_{p, q}^{1,2}$ and $\mathcal{F}$. Hence, $\mathcal{F}$ is a strong deformation retract. 
To prove (5.22), recall (5.15), (5.18) and (5.19), and consider that $\mathrm{d}_{x} \psi$ is an isometry and $\mathrm{d}_{x} \psi(x, t)[Y(x)]=Y(\psi(x, t))$. Setting $w=\mathcal{F}(x)$ and we compute directly:

$$
\begin{aligned}
\langle\dot{w}, \dot{w}\rangle-\langle\dot{x}, \dot{x}\rangle & =2 \phi^{\prime}\langle\dot{x}, Y\rangle+\left(\phi^{\prime}\right)^{2}\langle Y, Y\rangle= \\
& =\frac{\left(\int_{0}^{1} \frac{\langle\dot{x}, Y\rangle}{\langle Y, Y\rangle} \mathrm{d} s\right)^{2}\left(\int_{0}^{1} \frac{\mathrm{d} s}{\langle Y, Y\rangle}\right)^{-2}-\langle\dot{x}, Y\rangle^{2}}{\langle Y, Y\rangle} .
\end{aligned}
$$

Integrating on $[0,1]$, we obtain:

$$
2(J(w)-f(x))=\left(\int_{0}^{1} \frac{\langle\dot{x}, Y\rangle}{\langle Y, Y\rangle} \mathrm{d} s\right)^{2}\left(\int_{0}^{1} \frac{\mathrm{d} s}{\langle Y, Y\rangle}\right)^{-1}-\int_{0}^{1} \frac{\langle\dot{x}, Y\rangle^{2}}{\langle Y, Y\rangle} \mathrm{d} s .
$$

Hölder's inequality tells us that

$$
\left(\int_{0}^{1} \frac{\langle\dot{x}, Y\rangle}{\langle Y, Y\rangle} \mathrm{d} s\right)^{2} \leq\left(\int_{0}^{1} \frac{\langle\dot{x}, Y\rangle^{2}}{\langle Y, Y\rangle} \mathrm{d} s\right) \cdot\left(\int_{0}^{1} \frac{\mathrm{d} s}{\langle Y, Y\rangle},\right)
$$

and substituting in (5.23) gives

$$
J(w)-f(x) \geq 0 .
$$

Moreover, the equal sign in (5.24) holds precisely when $\langle\dot{x}, Y\rangle$ is constant, i.e. when $x \in \mathcal{N}_{p, q}$. This concludes the proof.

The proof of Theorem 1.3 is based on the following result of the classical Ljusternik-Schnirelman theory on infinite dimensional manifolds (see e.g. $[11,12])$ :

Theorem 5.10. Let $M$ be a Hilbert manifold and $F: M \longmapsto \mathbb{R}$ be a $C^{2}$ functional on $M$. Suppose that the following hypotheses are satisfied:

(1) $F$ is bounded from below;

(2) for all $c \geq \inf F, F$ satisfies the Palais-Smale condition at the level $c$;

(3) for all $c \geq \inf F$, the sublevel $F^{c}$ is a complete metric subspace of $M$.

Then, there exists at least cat ${ }_{M}(M)$ critical points of $F$ in $M$. Moreover, if cat $_{M}(M)=+\infty$, there exists a sequence $x_{n}$ of critical points of $F$ in $M$ such that:

$$
\lim _{n \rightarrow \infty} F\left(x_{n}\right)=\sup _{M} F
$$


Using Theorem 5.10, we can now prove Theorem 1.3:

Proof of Theorem 1.3. We show that the functional $J$ satisfies the hypothesis of Theorem 5.10.

By Proposition 3.1, $\mathcal{N}_{p, q}$ is a Hilbert manifold and $J$ is a $C^{2}$-functional on $\mathcal{N}_{p, q}$. By the pseudo-coercivity of $J$, Proposition 4.3 says that $J$ is bounded from below, and Theorem 5.3 gives the Palais-Smale condition for $J$ at every level $c \in \mathbb{R}$.

The completeness of the sublevels $J^{c}$ is proven in Proposition 5.5.

In order to conclude the proof, we only need to show that $J$ is unbounded from above in $\mathcal{N}_{p, q}$. From Proposition 5.9, it suffices to show that $f$ is unbounded from above in $\Omega_{p, q}^{1,2}$. To prove this, we use local coordinates as in the proof of Lemma 4.1 (see also Appendix C). We assume, without loss of generality, that $\mathcal{M}=U \times] a, b\left[\right.$, where $U$ is an open subset of $\mathbb{R}^{N-1}$, and, in the coordinates $\mathbf{x}=\left(x_{1}, \ldots, x_{N-1}\right) \in U$ and $\left.t \in\right] a, b$ [ the metric $g$ is written as in (4.2). We can also assume that the vector field $\delta$ and the scalar field $\beta$ are bounded in $U \times] a, b\left[\right.$. If we fix two points $p=\left(\mathbf{x}_{0}, t_{0}\right)$ and $q=\left(\mathbf{x}_{1}, t_{1}\right)$ in $U \times] a, b[$, we choose a smooth function $t:[0,1] \longmapsto] a, b\left[\right.$ with $t(0)=t_{0}$ and $t(1)=t_{1}$. We can find a sequence of smooth curves $\mathbf{x}_{k}:[0,1] \longmapsto U$, $k \geq 1$, joining $\mathbf{x}_{0}$ and $\mathbf{x}_{1}$, such that $\left\langle\dot{\mathbf{x}}_{k}, \dot{\mathbf{x}}_{k}\right\rangle_{0}$ diverges uniformly on $[0,1]$ as $k \rightarrow \infty$. Setting $z_{k}=\left(\mathbf{x}_{k}, t\right) \in \Omega_{p, q}^{1,2}$, since $\delta$ and $\beta$ are bounded it follows immediately that $\lim _{k \rightarrow \infty} f\left(z_{k}\right)=+\infty$, and we are done.

\section{Multiplicity of Timelike Geodesics.}

The purpose of this Section is to prove Theorem 1.4. We will assume henceforth that all the hypotheses of Theorem 1.4 are satisfied.

We will work with the spaces $\mathcal{N}_{p, \gamma_{q}(t)}$, and, with a slight abuse of notations, we will denote by $J$ the restriction of the action functional $f$ on each of them. To avoid confusion, for every $c, t \in \mathbb{R}$, we will denote by $J^{c} \cap \mathcal{N}_{p, \gamma_{q}(t)}$ the $c$-sublevel of the functional $J$ in $\mathcal{N}_{p, \gamma_{q}(t)}$.

For all $t \in \mathbb{R}$, we define a map $\mathcal{L}_{t}$ between the spaces $\mathcal{N}_{p, q}$ and $\mathcal{N}_{p, \gamma_{q}(t)}$ as follows.

For $x \in \mathcal{N}_{p, q}$, let $C_{x}$ denote the constant $\langle\dot{x}, Y\rangle$, and $\mathcal{L}_{t}(x)=w$ the curve:

$$
w(s)=\psi\left(x(s), \phi_{t}(s)\right)
$$


where $\psi(\cdot, \cdot)$ is the flow of $Y$, and $\phi_{t}$ is the function:

$$
\phi_{t}(s)=t \cdot\left(\int_{0}^{1} \frac{\mathrm{d} r}{\langle Y, Y\rangle}\right)^{-1} \int_{0}^{s} \frac{\mathrm{d} r}{\langle Y, Y\rangle}
$$

Since $\phi_{t}(0)=0$ and $\phi_{t}(1)=t$, it is clear that $\mathcal{L}_{t}(x) \in \Omega_{p, \gamma_{q}(t)}^{1,2}$. Moreover, using the fact that $\mathrm{d}_{x} \psi$ is an isometry, $\mathrm{d}_{x} \psi[Y(x)]=Y(w)$ and $\mathrm{d}_{t} \psi=Y$, we compute directly:

$$
\langle\dot{w}, Y\rangle=\left\langle\mathrm{d}_{x} \psi[\dot{x}], Y\right\rangle+\phi_{t}^{\prime} \cdot\langle Y, Y\rangle=C_{x}+t \cdot\left(\int_{0}^{1} \frac{\mathrm{d} r}{\langle Y, Y\rangle}\right)^{-1} .
$$

So, the quantity

$$
C_{w} \equiv\langle\dot{w}, Y\rangle=C_{x}+t \cdot\left(\int_{0}^{1} \frac{\mathrm{d} r}{\langle Y, Y\rangle}\right)^{-1}
$$

is constant and $\mathcal{L}_{t}(x) \in \mathcal{N}_{p, \gamma_{q}(t)}$.

The map $\mathcal{L}_{t}$ satisfies the following properties:

Proposition 6.1. Let $t$ be a real number and $\mathcal{L}_{t}: \mathcal{N}_{p, q} \longmapsto \mathcal{N}_{p, \gamma_{q}(t)}$ be the map described above. Then, the following are true:

(1) $\mathcal{L}_{t}$ is a bijection;

(2) $\mathcal{L}_{t}$ is a map of class $C^{2}$;

(3) for all compact subset $B \subset \mathcal{N}_{p, q}$ it is

$$
\lim _{|t| \rightarrow+\infty} \sup _{x \in B} J\left(\mathcal{L}_{t}(x)\right)=-\infty .
$$

Proof. For part (1) it is enough to observe that, since $\gamma_{\gamma_{q}(t)}(-t)=q$ and the quantity $\langle Y, Y\rangle$ is constant along the flow lines of $Y$, then the map

$$
\mathcal{F}_{-t}: \mathcal{N}_{p, \gamma_{q}(t)} \longmapsto \mathcal{N}_{p, q}
$$

is an inverse for $\mathcal{L}_{t}$.

For part (2), observe that, from (6.2), $\phi_{t}$ depends regularly on $x$. 
For part (3), we denote by $w=\mathcal{L}_{t}(x)$. Using (6.2) and (6.3) we compute $J(w)$ as follows:

$$
\begin{aligned}
J(w) & =\frac{1}{2} \int_{0}^{1}\langle\dot{w}, \dot{w}\rangle \mathrm{d} s= \\
& =\frac{1}{2} \int_{0}^{1}\left(\left\langle\mathrm{~d}_{x} \psi[\dot{x}], \mathrm{d}_{x} \psi[\dot{x}]\right\rangle+2 \phi_{t}^{\prime}\left\langle\mathrm{d}_{x} \psi[\dot{x}], Y\right\rangle+\left(\phi_{t}^{\prime}\right)^{2}\langle Y, Y\rangle\right) \mathrm{d} s=
\end{aligned}
$$

$$
\begin{aligned}
& =\frac{1}{2} \int_{0}^{1}\left(\langle\dot{x}, \dot{x}\rangle+2 \phi_{t}^{\prime} C_{x}+\left(\phi_{t}^{\prime}\right)^{2}\langle Y, Y\rangle\right) \mathrm{d} s= \\
& =\frac{1}{2} \int_{0}^{1}\langle\dot{x}, \dot{x}\rangle \mathrm{d} s+\frac{C_{w}^{2}-C_{x}^{2}}{2} \int_{0}^{1} \frac{\mathrm{d} s}{\langle Y, Y\rangle}= \\
& =J(x)+\frac{C_{w}^{2}-C_{x}^{2}}{2} \int_{0}^{1} \frac{\mathrm{d} s}{\langle Y, Y\rangle} .
\end{aligned}
$$

If $B$ is a compact subset of $\mathcal{N}_{p, q}$, then there exist positive constants $D=$ $D(B), \mu=\mu(b)$, and $\nu=\nu(B)$ such that for all $x \in B$ it is $\left|C_{x}\right| \leq D$ and $-\mu \leq\langle Y(x(s)), Y(x(s))\rangle \leq-\nu$. Hence, from (6.4) we obtain:

$$
J(w) \leq c+\frac{D^{2}}{2 \nu}-\frac{C_{w}^{2}}{2 \mu} .
$$

In order to conclude the proof, it suffices to show that $C_{w}=C_{w}(x, t)$ can be made arbitrarily large as $|t| \rightarrow+\infty$, uniformly in $x$. This follows immediately from (6.3), that gives:

$$
\left|C_{w}\right| \geq|t| \int_{0}^{1} \frac{-\mathrm{d} s}{\langle Y, Y\rangle}-\left|C_{x}\right| \geq \frac{|t|}{\mu}-D .
$$

We can now prove Theorem 1.4.

Proof of Theorem 1.4. Since $\mathcal{M}$ is non contractible, a well known result of Fadell and Husseini (see [7]) says that there exists a sequence $\left\{K_{n}\right\}_{n \in}$ of compact subsets of $\Omega_{p, q}^{1,2}$ such that:

$$
\lim _{n \rightarrow \infty} \operatorname{cat}_{\Omega_{p, q}^{1,2}}\left(K_{n}\right)=+\infty .
$$

Let $\mathcal{F}: \Omega_{p, q}^{1,2} \longmapsto \mathcal{N}_{p, q}$ be the strong deformation retract described in Section 5 , and consider the compact subsets of $\mathcal{N}_{p, q}$ :

$$
\tilde{K}_{n}=\mathcal{F}\left(K_{n}\right), n \in \mathbb{N} \text {. }
$$


Since $\mathcal{F}$ is an homotopy equivalence, then:

$$
\operatorname{cat}_{\mathcal{N}_{p, q}}\left(\tilde{K}_{n}\right)=\operatorname{cat}_{\Omega_{p, q}^{1,2}}\left(K_{n}\right) \longmapsto+\infty .
$$

Let $c_{0}<0$ and $t_{0}>0$ be chosen as in the statement of Theorem 1.4 and fix $c<c_{0}$. From part (3) of Proposition 6.1, it follows that, for every $m \in \mathbb{N}$, there exists a positive number $\bar{t}=\bar{t}(m) \geq t_{0}$ such that the first $m$ elements of the sequence:

$$
\mathcal{L}_{t}\left(\tilde{K}_{1}\right), \mathcal{L}_{t}\left(\tilde{K}_{2}\right), \ldots, \mathcal{L}_{t}\left(\tilde{K}_{m}\right)
$$

are contained in the sublevel $J^{c} \cap \mathcal{N}_{p, \gamma_{q}(t)}$ for all $t$ with $|t| \geq \bar{t}$.

For every $t \in \mathbb{R}$, since $\mathcal{L}_{t}: \mathcal{N}_{p, q} \longmapsto \mathcal{N}_{p, \gamma_{q}(t)}$ is a homeomorphism, then $\mathcal{L}_{t}\left(\tilde{K}_{i}\right)$ is a compact subset of $\mathcal{N}_{p, \gamma_{q}(t)}$ and

$$
\operatorname{cat}_{\mathcal{N}_{p, \gamma_{q}(t)}}\left(\mathcal{L}_{t}\left(\tilde{K}_{i}\right)\right)=\operatorname{cat}_{\mathcal{N}_{p, q}}\left(\tilde{K}_{i}\right), \quad i=1,2, \ldots, m .
$$

Let $M(m)=\max \left\{\operatorname{cat}_{\mathcal{N}_{p, \gamma_{q}(t)}}\left(\mathcal{L}_{t}\left(\tilde{K}_{i}\right)\right): i=1,2, \ldots, m\right\}$. Clearly, $M(m)$ tends to $+\infty$ as $m \rightarrow+\infty$.

For $j \in \mathbb{N}$, we denote by $\mathcal{A}_{t}(j)$ the set of closed subset of $\mathcal{N}_{p, \gamma_{q}(t)}$ with Ljusternik-Schnirelman category greater or equal to $j$. A classical minimax argument in Critical Point Theory for Palais-Smale functionals (see e.g. [11]) show that, for $j=1,2, \ldots, M(m)$, the numbers:

$$
c_{j}=\inf _{A \in \mathcal{A}_{t}(j)}\left[\sup _{x \in A} J(x)\right] \leq c<0,
$$

are critical values for $J$ on $\mathcal{N}_{p, \gamma_{q}(t)}$. If the $c_{i}$ 's are not all distinct, and there exist $i \neq j$ such that $c_{i}=c_{j}$, then there are infinitely many critical points for $J$ at the level $c_{i}$. If they are all distinct, then there are $M(m)$ distinct critical points for $J$. Hence, if $|t| \rightarrow+\infty$, the number of timelike geodesics in $\mathcal{N}_{p, \gamma_{q}(t)}$ is arbitrarily large, and we are done.

Remark 6.2. It is easy to check that, for all $t \in \mathbb{R}$, the map $\mathcal{G}_{t}$ : $\mathcal{N}_{\gamma_{p}(-t), q} \longmapsto \mathcal{N}_{p, \gamma_{q}(t)}$ defined by $\mathcal{G}_{t}(x)=w$, with $w(s)=\psi(x(s), t)$ satisfies the properties:

(a) $\mathcal{G}_{t}$ is a bijective isometry;

(b) $J\left(\mathcal{G}_{t}(x)\right)=J(x)$, for all $x \in \mathcal{N}_{\gamma_{p}(-t), q}$.

It follows immediately that if $f$ is $c$-precompact in $\mathcal{N}_{p, \gamma_{q}(t)}$, then it is $c$ precompact also in $\mathcal{N}_{\gamma_{p}(-t), q}$. 


\section{A. Examples and Remarks on the Hypotheses of Theorem 1.2.}

The primary goal of this section is to give examples where the hypotheses of Theorems 1.2, 1.3 and 1.4 are satisfied.

We recall that the Lorentzian gradient $\nabla \Psi$ of a smooth real function $\Psi$ defined on (an open subset of) $\mathcal{M}$ is the vector field $\nabla \Psi$ defined by the relation $\mathrm{d} \Psi(z)[v]=\langle\nabla \Psi(z), v\rangle$ for all $z$ and all $v \in T_{z} \mathcal{M}$. A smooth function is said to be a time function if its gradient is timelike everywhere.

In this Section we will assume that $\mathcal{M}$ is the domain of a smooth time function $T$ satisfying:

$$
\langle\nabla T, Y\rangle \equiv 1
$$

It is easy to see that such a time function $T$ always exists locally (see Appendix C, (C.8)). Observe also that the condition (A.1) is equivalent to:

$$
T(\psi(x, t))=T(x)+t, \quad \forall x \in \mathcal{M}, t \in \mathbb{R},
$$

which means that the flow lines of the vector field $Y$ are parametrized by the time function $T$. Observe also that, if $Y$ is complete, then a necessary and sufficient condition for such a function to exist is that $\mathcal{M}$ admit a spacelike surface $\Sigma$ that intersects exactly once all the flow lines of $Y$.

We denote by $\Delta_{0}=\nabla T^{\perp}$ the orthogonal distribution to the vector field $\nabla T$ on $\mathcal{M}$. Equivalently, $\Delta_{0}$ is the integrable distribution on $\mathcal{M}$ given by the tangent spaces to the spacelike surfaces $T=T_{0}$. By (A.2), these surfaces are preserved by the flow of $Y$, hence $\Delta_{0}$ is a $Y$-invariant, integrable spacelike distribution of codimension 1 on $\mathcal{M}$.

We now fix some notation needed to introduce the concept of spatial sublinear growth for vector fields on $\mathcal{M}$.

We denote by $\Sigma$ the spacelike hypersurface of $\mathcal{M}$ given by $T^{-1}(0)$; we denote by $\mathrm{d}_{\Sigma}$ the Riemannian distance function on $\Sigma$ induced by the restriction of the metric of $\mathcal{M}$ on $\Sigma$.

Since $\Sigma$ intersects exactly once the flow lines of $Y$, then, for every point $x \in \mathcal{M}$ it is well defined the projection $\mathbf{P}(x)$ of $x$ on $\Sigma$, given by:

$$
\mathbf{P}(x)=\Sigma \cap \gamma_{x},
$$

where $\gamma_{x}$ is the maximal integral curve of $Y$ through $x$. Finally, we introduce the spatial pseudo-distance function $\mathrm{d}_{\sigma}$ on $\mathcal{M}$, given by:

$$
\mathrm{d}_{\sigma}\left(x_{0}, x_{1}\right)=\mathrm{d}_{\Sigma}\left(\mathbf{P}\left(x_{0}\right), \mathbf{P}\left(x_{1}\right)\right) .
$$

Roughly speaking, $\mathrm{d}_{\sigma}$ measures the distance between the flow lines of $Y$. 
Definition A.1. A function $b: \mathcal{M} \longmapsto \mathbb{R}$ is said to have spatial sublinear growth on $\mathcal{M}$ if there exists $p_{0} \in \mathcal{M}$, a constant $\left.\alpha \in\right] 0,1[$ and two constants $A, B \in \mathbb{R}$ such that, for all $x \in \mathcal{M}$ it is:

$$
|b(x)| \leq A \cdot \mathrm{d}_{\sigma}\left(x, p_{0}\right)^{\alpha}+B .
$$

A vector field $W$ on $\mathcal{M}$ is said to have spatial sublinear growth on $\mathcal{M}$ if, for every $x \in \mathcal{M}$ and every $\xi \in\left(\Delta_{0}\right)_{x}$ it is:

$$
|\langle W, \xi\rangle| \leq b(x) \cdot \sqrt{\langle\xi, \xi\rangle},
$$

for some function $b$ having spatial sublinear growth on $\mathcal{M}$.

Obviously, since $\alpha \in] 0,1\left[\right.$, the choice of the base point $p_{0}$ in (A.3) is purely formal.

We give the following integral characterization of the spatial sublinear condition for a vector field $W$ :

Lemma A.2. Let $z:[0,1] \longmapsto \mathcal{M}$ be an absolutely continuous curve, and, for almost every $s$, set $\dot{z}(s)=\xi(s)+\lambda(s) \cdot Y(z(s))$, where $\xi(s) \in\left(\Delta_{0}\right)_{z(s)}$ and $\lambda(s) \in \mathbb{R}$. If $W$ is a continuous vector field having spatial sublinear growth on $\mathcal{M}$, then there exists a continuous function $\chi: \mathbb{R}^{+} \longmapsto \mathbb{R}^{+}$, depending only on the initial point $z(0)$, such that:

$$
\int_{0}^{1}|\langle W, \xi\rangle| \mathrm{d} s \leq \chi\left(\int_{0}^{1} \sqrt{\langle\xi, \xi\rangle} \mathrm{d} s\right)
$$

where $\chi(r)$ has order of infinity less than 2 for $r \rightarrow+\infty$, i.e.,

$$
\limsup _{r \rightarrow+\infty} \frac{\chi(r)}{r^{2}}=0
$$

Proof. Let $x$ denote the projection of $z$ on $\Sigma$, i.e., $x(s)=\mathbf{P}(x(s))$ for all $s$. Since $Y$ is Killing, it is:

$$
\langle\dot{x}(s), \dot{x}(s)\rangle=\langle\xi(s), \xi(s)\rangle, \text { for almost all } s .
$$

By the spatial boundedness of $W$, we can assume without loss of generality that

$$
|\langle W(z(s)), \xi(s)\rangle| \leq\left[A \cdot \mathrm{d}_{\sigma}(z(s), x(0))^{\alpha}+B\right] \cdot \sqrt{\langle\xi(s), \xi(s)\rangle}
$$


for some real constants $A, B$ and some $\alpha \in] 0,1[$. Moreover, from the definition of $\mathrm{d}_{\sigma}$, we have:

$$
\mathrm{d}_{\sigma}(z(s), x(0)) \leq \int_{0}^{s} \sqrt{\langle\dot{x}, \dot{x}\rangle} \mathrm{d} r \leq \int_{0}^{1} \sqrt{\langle\xi, \xi\rangle} \mathrm{d} s .
$$

Integrating (A.5) on $[0,1]$, using Schwartz's inequality and substituting (A.6), we obtain:

$$
\int_{0}^{1}|\langle W, \xi\rangle| \mathrm{d} s \leq A \cdot r^{\alpha+1}+B \cdot r^{\frac{1}{2}}
$$

where $r=\int_{0}^{1} \sqrt{\langle\xi, \xi\rangle} \mathrm{d} s$. This concludes the proof.

The spatial sublinear growth for the vector field $Y$ implies the $c$-precompactness of $\mathcal{N}_{p, q}$, as explained in the following Proposition:

Proposition A.3. Suppose that $\mathcal{M}$ is connected and complete with respect to $g_{(\mathrm{R})}$, and that $Y$ satisfies the boundedness assumption (4.11) in $\mathcal{M}$. If $Y$ has spatial sublinear growth in $\mathcal{M}$, then $\mathcal{N}_{p, q}$ is c-precompact for every pair of $(p, q)$ and every $c \in \mathbb{R}$.

Proof. Let $p, q \in \mathcal{M}$ and $c \in \mathbb{R}$ be fixed and set $\vartheta=T(q)-T(p)$.

Let $z \in J^{c}$ be a fixed curve; for almost all $s$ we decompose the tangent vector $\dot{z}(s)$ as the sum of a vector $\xi(s)$ in $\left(\Delta_{0}\right)_{z(s)}$ and a multiple of $Y(z(s))$. Recalling (A.1), an immediate calculation gives:

$$
\dot{z}(s)=\xi(s)+\langle\dot{z}(s), \nabla T(z(s))\rangle \cdot Y(z(s)), \quad \xi(s) \in\left(\Delta_{0}\right)_{z(s)} .
$$

Setting $C_{z}=\langle\dot{z}, Y\rangle$, we have

$$
C_{z}=\langle\xi, Y\rangle+\langle\dot{z}, \nabla T\rangle \cdot\langle Y, Y\rangle
$$

and, integrating on $[0,1]$, we obtain

$$
\vartheta=\int_{0}^{1}\langle\dot{z}, \nabla T\rangle \mathrm{d} s=C_{z} \cdot \int_{0}^{1} \frac{\mathrm{d} s}{\langle Y, Y\rangle}-\int_{0}^{1} \frac{\langle\xi, Y\rangle}{\langle Y, Y\rangle} \mathrm{d} s
$$

which gives:

$$
C_{z}=\left(\vartheta+\int_{0}^{1} \frac{\langle\xi, Y\rangle}{\langle Y, Y\rangle} \mathrm{d} s\right) \cdot\left(\int_{0}^{1} \frac{\mathrm{d} s}{\langle Y, Y\rangle}\right)^{-1} .
$$


Now, we write the product $\langle\dot{z}, \dot{z}\rangle$ as:

$$
\langle\dot{z}, \dot{z}\rangle=\langle\xi, \xi\rangle-\frac{\langle\xi, Y\rangle^{2}}{\langle Y, Y\rangle}+\frac{C_{z}^{2}}{\langle Y, Y\rangle} .
$$

Now, using the Hölder's inequality gives :

$$
\left(\int_{0}^{1} \frac{\langle\xi, Y\rangle}{\langle Y, Y\rangle} \mathrm{d} s\right)^{2} \leq\left(\int_{0}^{1} \frac{\langle\xi, Y\rangle^{2}}{\langle Y, Y\rangle} \mathrm{d} s\right) \cdot\left(\int_{0}^{1} \frac{\mathrm{d} s}{\langle Y, Y\rangle}\right) .
$$

Then, integrating (A.8) on $[0,1]$ and using (A.7), we get:

$$
\begin{aligned}
2 c & \geq \int_{0}^{1}\langle\dot{z}, \dot{z}\rangle \mathrm{d} s \geq \\
& \geq \int_{0}^{1}\langle\xi, \xi\rangle \mathrm{d} s+2 \vartheta\left(\int_{0}^{1} \frac{\mathrm{d} s}{\langle Y, Y\rangle}\right)^{-1} \int_{0}^{1} \frac{\langle\xi, Y\rangle}{\langle Y, Y\rangle} \mathrm{d} s+\vartheta^{2}\left(\int_{0}^{1} \frac{\mathrm{d} s}{\langle Y, Y\rangle}\right)^{-1} .
\end{aligned}
$$

Finally, using (4.11), from (A.9) we obtain:

$$
\int_{0}^{1}\langle\xi, \xi\rangle \mathrm{d} s \leq A_{1} \cdot \int_{0}^{1}|\langle\xi, Y\rangle| \mathrm{d} s+B_{1},
$$

for some real constants $A_{1}, B_{1}$ that are independent from $z$. Since $Y$ has spatial sublinear growth, from Lemma A.2 it follows that the integral

$$
\int_{0}^{1}\langle\xi, \xi\rangle \mathrm{d} s
$$

is bounded independently of $z$, hence, by (A.10), also the integral

$$
\int_{0}^{1}|\langle\xi, Y\rangle| \mathrm{d} s
$$

is bounded independently of $z$. From (4.11) and (A.7), it follows that $\left|C_{z}\right|$ is bounded in the sublevel $J^{c}$. Since

$$
\int_{0}^{1}\langle\dot{z}, \dot{z}\rangle_{(\mathrm{R})} \mathrm{d} s=J(z)-2 C_{z}^{2} \int_{0}^{1} \frac{\mathrm{d} s}{\langle Y, Y\rangle}
$$

is bounded, then the Ascoli-Arzelá Theorem implies that $\mathcal{N}_{p, q}$ is $c$-precompact. This concludes the proof. 
As an example, consider the manifold $\mathcal{M}$ given by a global splitting $\mathcal{M}_{0} \times \mathbb{R}$, described by coordinates $\mathbf{x} \in \mathcal{M}_{0}$ and $t \in \mathbb{R}$, where $\left(\mathcal{M}_{0},\langle\cdot, \cdot\rangle_{0}\right)$ is a complete Riemannian manifold, and Lorentzian metric $g$ given by:

$$
g(\mathbf{x}, t)[(\xi, \tau),(\xi, \tau)]=\langle\xi, \xi\rangle_{0}+2\langle\delta(\mathbf{x}), \dot{\mathbf{x}}\rangle-\beta(\mathbf{x}) \tau^{2}, \quad \xi \in T_{\mathbf{x}} \mathcal{M}_{0}, \tau \in \mathbb{R},
$$

where $\delta$ is a smooth vector field on $\mathcal{M}_{0}$ and $\beta: \mathcal{M}_{0} \longmapsto \mathbb{R}^{+}$is a smooth scalar field. Let $Y$ be the timelike Killing vector field $(\mathbf{0}, 1)=\frac{\partial}{\partial t}$. The timelike vector field:

$$
W(\mathbf{x}, t)=\frac{1}{\beta(\mathbf{x})+\langle\delta(\mathbf{x}), \delta(\mathbf{x})\rangle_{0}} \cdot(\delta(\mathbf{x}),-1),
$$

is integrable (see Appendix C, (C.6)) and it is easily checked that $\langle Y, W\rangle \equiv 1$. Here, the orthogonal distribution $\Delta_{0}=W^{\perp}$ is identified with the tangent bundle of $\mathcal{M}_{0}$, and, for $(\mathbf{x}, \xi) \in T \mathcal{M}_{0}$, it is

$$
\langle Y, \xi\rangle^{2}=\langle\delta(\mathbf{x}), \xi\rangle_{0}^{2} \leq\langle\delta(\mathbf{x}), \delta(\mathbf{x})\rangle_{0} \cdot\langle\xi, \xi\rangle_{0}=\langle\delta(\mathbf{x}), \delta(\mathbf{x})\rangle_{0} \cdot\langle\xi, \xi\rangle .
$$

Moreover, $\langle Y, Y\rangle=-\beta$. In this case, if $\beta$ is bounded from above and bounded away from zero, and if $\|\delta\|_{0}=\sqrt{\langle\delta(\mathbf{x}), \delta(\mathbf{x})\rangle_{0}}$ has sublinear growth in $\mathcal{M}_{0}$, then $Y$ has spatial sublinear growth in $\mathcal{M}$, and the hypothesis of Proposition A.3 are satisfied. By Theorem 1.2, $\mathcal{M}$ is geodesically connected. Thus, the results of [6] and [8] follow from ours.

We recall that a $C^{1}$-vector field $W$ is said to be irrotational if its curl vanishes on the orthogonal distribution of $W$. The curl of $W$ is the differential $\mathrm{d} \omega_{W}$ of the dual form $\omega_{W}(v)=\langle W, v\rangle$.

Remark A.4. Observe that, if $\mathcal{M}$ is static with respect to $Y$, i.e. the Killing vector field $Y$ is irrotational, then the orthogonal distribution of $Y$ is integrable (see Proposition 12.30 of [13]). In this case, the condition of spatial sublinear growth is automatically satisfied by $Y$ by taking $\Delta_{0}=Y^{\perp}$.

Remark A.5. We emphasize the fact that the Riemannian completeness for $\mathcal{M}$ assumed in Proposition A.3 is, in general, not related to the Lorentzian completeness, nor to the geodesical connectedness of $\mathcal{M}$. Some examples that prove the logical inequivalence of these concepts may be found in $[2,3]$.

\section{B. Pseudo-Coercivity and Global Hyperbolicity.}

In this appendix we discuss some relations of the pseudo-coercivity property and the global hyperbolicity for $\mathcal{M}$. We recall the basic notions needed; 
our main reference for this part is the book by B. O'Neill ([13]).

A regular (absolutely continuous) curve $\gamma:] a, b[\subseteq \mathbb{R} \longmapsto \mathcal{M}$ is called timelike (resp. lightlike, spacelike, causal) if $\dot{\gamma}(s)$ is timelike (resp. lightlike, spacelike, causal) for all $s \in] a, b[$ (almost everywhere in $] a, b[$ ). A Lorentzian manifold $\mathcal{M}$ is said to be causal if it does not contain any closed causal curve. The manifold $\mathcal{M}$ is strongly causal if given any point $p \in \mathcal{M}$ and any neighborhood $V$ of $p$ there exists a neighborhood $U \subset V$ of $p$ such that all the causal curves with endpoints in $U$ remain inside $V$.

For a pair $p, q$ of points of $\mathcal{M}$, the causality relation $p<q$ means that there exists a future pointing causal curve from $p$ to $q$. Two points $p$ and $q$ are causally related if either $p<q$ or $q<p$. The relation $p \leq q$ means that either $p<q$ or $p=q$. The causal future $\mathcal{J}^{+}(p)$ and the causal past $\mathcal{J}^{-}(p)$ of $p$ are the sets:

$$
\mathcal{J}^{+}(p)=\{q \in \mathcal{M}: p \leq q\}, \quad \mathcal{J}^{-}(p)=\{q: q \leq p\} .
$$

Finally, the manifold $\mathcal{M}$ is said to be globally hyperbolic if it is strongly causal and if, for every pair of points $p, q \in \mathcal{M}$ the set $\mathcal{J}^{+}(p) \cap \mathcal{J}^{-}(q)$ is compact in $\mathcal{M}$. Equivalently, if $\mathcal{M}$ is strongly causal, then it is globally hyperbolic if for every pair of causally related points $p<q$ and every sequence $z_{n}$ of future pointing causal curves from $p$ to $q$ there exists a subsequence $z_{n_{k}}$ which is uniformly convergent, up to a reparametrization.

Our pseudo-coercivity assumption implies the global hyperbolicity, as proven in the following:

Proposition B.1. If $f$ is pseudo-coercive on $\mathcal{C}_{p, q}$ for all pairs $p, q$ in $\mathcal{M}$, then $\mathcal{M}$ is globally hyperbolic.

Nevertheless, the global hyperbolicity is in general not sufficient to guarantee the geodesical connectedness, not even for stationary manifolds.

To see this, let's consider the following example. Let $\mathcal{M}$ be the 4dimensional Minkowski space, with $Y=\frac{\partial}{\partial t}$ the timelike Killing vector field. Take a non convex open subset $A$ of the spacelike surface $t=0$, and consider the Cauchy development $D(A)$ of $A$, which is the set of points $p$ in $\mathcal{M}$ such that every past or future pointing, inextendible causal curve through $p$ meets $A$ (see Definition 14.45 of [13]). The interior of $D(A)$ is non empty, as it contains $A$, and so by Theorem 14.38 of [13] it is a globally hyperbolic manifold. Nonetheless, it is not geodesically connected, because two points in $A$ cannot joined by any geodesics contained in $D(A)$. 
Proof of Proposition B.1. We assume that $f$ is pseudo-coercive in $\mathcal{N}_{p, q}$ for all $p$ and $q$ in $\mathcal{M}$. Suppose that the strong causality of $\mathcal{M}$ is violated; in this case there exist a point $p \in \mathcal{M}$, a neighborhood $V$ of $p$ and a sequence $z_{n}:[0,1] \longmapsto \mathcal{M}$ of causal curves such that:

(a) $p_{n} \equiv z_{n}(0)$ and $q_{n} \equiv z_{n}(1)$ tend to $p$ as $n \rightarrow \infty$;

(b) $z_{n}([0,1]) \cap(\mathcal{M} \backslash V) \neq \emptyset$ for all $n$.

Since $z_{n}$ is causal, we can reparameterize each $z_{n}$ and suppose that $\left\langle\dot{z}_{n}, Y\right\rangle=$ $C_{n}$ is constant for all $n$.

We can find two sequences of curves $x_{n}^{1}, x_{n}^{2}:[0,1] \longmapsto V, n \in \mathbb{N}$, satisfying

$$
x_{n}^{1}(0)=p, x_{n}^{1}(1)=p_{n}, x_{n}^{2}(0)=q_{n}, x_{n}^{2}(1)=q,
$$

and such that $\dot{x}_{n}^{i}$ tends to 0 uniformly in [0,1], $i=1,2$. For instance, one can take the minimal geodesics with respect to $g_{(\mathrm{R})}$ that join $p$ with $p_{n}$ and $q_{n}$ respectively.

Let $y_{n}:[0,1] \longmapsto \mathcal{M}$ be the junction of $x_{n}^{1}, z_{n}$ and $x_{n}^{2}$, defined by $y_{n}(s)=x_{n}^{1}(3 s)$ if $s \in\left[0, \frac{1}{3}\right], y_{n}(s)=z_{n}(3 s-1)$ if $s \in\left[\frac{1}{3}, \frac{2}{3}\right]$ and $y_{n}(s)=$ $x_{n}^{2}(3 s-2)$ if $s \in\left[\frac{2}{3}, 1\right]$. Now, define $w_{n}=\mathcal{F}\left(y_{n}\right)$, where $\mathcal{F}: \Omega_{p, p}^{1,2} \longmapsto \mathcal{N}_{p, p}$ is the map defined in section 5 . Notice that, since $\left\langle\dot{z}_{n}, Y\right\rangle$ is constant, then $w_{n}(s)=z_{n}(3 s-1)$ for $\left.s \in\right] \frac{1}{3}, \frac{2}{3}[$.

Since $\dot{x}_{n}^{i}$ is uniformly convergent to 0 , by the construction of the map $\mathcal{F}$ one sees that:

$$
J\left(w_{n}\right) \leq \varepsilon_{n}
$$

where $\varepsilon_{n}$ is a sequence in $\mathbb{R}$ that converges to 0 . By taking a re-indexed subseqence, we can assume that $J\left(w_{n}\right) \leq \frac{1}{n}$.

Finally, define $\tilde{w}_{n}(s)=w_{n}(n s-k)$ for $s \in\left[\frac{k}{n}, \frac{k+1}{n}\right]$, where $k=0,1, \ldots, n-1$. By construction, it is $\tilde{w}_{n} \in \mathcal{N}_{p, q}$ and $J\left(\tilde{w}_{n}\right) \leq 1$. But, by (2) above, $\tilde{w}_{n}$ does not have any uniformly convergent subsequence, contradicting the 1precompactness of $\mathcal{N}_{p, p}$. Hence, $\mathcal{M}$ is strongly causal.

Now, fix any pair $p$ and $q$ of causally related points in $\mathcal{M}$ and consider any sequence $z_{n}$ of future pointing timelike curves in $\mathcal{M}$ joining $p$ and $q$. Since $\left\langle\dot{z}_{n}, Y\right\rangle<0$ everywhere, they can be reparameterized in such a way that $\left\langle\dot{z}_{n}, Y\right\rangle$ is constant. As $J\left(z_{n}\right) \leq 0$, by the pseudo-coercivity $\left\{z_{n}\right\}$ must contain a uniformly convergent subsequence. This implies that $\mathcal{M}$ is globally hyperbolic and we are done. 


\section{About the Local Structure of $\mathcal{M}$.}

For the reader's convenience, in this Appendix we collect a few results about the local structure of a Lorentzian manifold that admits a timelike Killing vector field, and that were used at some points during the rest of the paper. We claim no originality of the results and the proofs presented, that are mostly well known among specialists.

We assume that $\mathcal{M}$ is a Lorentzian manifold of dimension $N \geq 2$, and that $Y$ is a given timelike Killing vector field on $\mathcal{M}$.

Since $Y$ never vanishes on $\mathcal{M}$, then for every point $z \in \mathcal{M}$ there exists an open neighborhood $U_{z}$ of $z$ and a coordinate system $\varphi=\left(x_{1}, x_{2}, \ldots, x_{N-1}, t\right)$ on $U_{z}$, such that $Y$ is a coordinate field with respect to $\varphi$, say:

$$
Y=\frac{\partial}{\partial t} \text {. }
$$

We set $x_{N}=t$. It is easy to prove that the Killing property of $Y$ is expressed by the fact that, in such a coordinate system, the coefficients $g_{i j}$ of the metric $g$ on $\mathcal{M}$ are functions that do not depend on the variable $t$ :

Lemma C.1. For every coordinate system $\left(U_{z}, \varphi\right)$ around $z$ such that (C.1) holds, the coefficients $g_{i j}$ are independent of the variable $t$.

Proof. It is a simple calculation that uses the properties of the Levi-Civita connection of $g$. Namely, for every $i, j \in\{1,2, \ldots, N\}$ it is

$$
\begin{aligned}
\frac{\partial g_{i j}}{\partial t}= & \frac{\partial}{\partial t}\left\langle\frac{\partial}{\partial x_{i}}, \frac{\partial}{\partial x_{j}}\right\rangle= \\
= & \left\langle\nabla_{\frac{\partial}{\partial t}} \frac{\partial}{\partial x_{i}}, \frac{\partial}{\partial x_{j}}\right\rangle+\left\langle\frac{\partial}{\partial x_{i}}, \nabla_{\frac{\partial}{\partial t}} \frac{\partial}{\partial x_{j}}\right\rangle= \\
= & \left\langle\nabla_{\frac{\partial}{\partial x_{i}}} \frac{\partial}{\partial t}, \frac{\partial}{\partial x_{j}}\right\rangle+\left\langle\left[\frac{\partial}{\partial t}, \frac{\partial}{\partial x_{i}}\right], \frac{\partial}{\partial x_{j}}\right\rangle+ \\
& +\left\langle\frac{\partial}{\partial x_{i}}, \nabla_{\frac{\partial}{\partial x_{j}}} \frac{\partial}{\partial t}\right\rangle+\left\langle\frac{\partial}{\partial x_{i}},\left[\frac{\partial}{\partial t}, \frac{\partial}{\partial x_{j}}\right]\right\rangle .
\end{aligned}
$$

Since the $\frac{\partial}{\partial x_{i}}$ 's are coordinate fields, then $\left[\frac{\partial}{\partial x_{i}}, \frac{\partial}{\partial t}\right]=\left[\frac{\partial}{\partial t}, \frac{\partial}{\partial x_{j}}\right]=0$; moreover, by (1.6):

$$
\left\langle\nabla_{\frac{\partial}{\partial x_{i}}} \frac{\partial}{\partial t}, \frac{\partial}{\partial x_{j}}\right\rangle+\left\langle\frac{\partial}{\partial x_{i}}, \nabla_{\frac{\partial}{\partial x_{j}}} \frac{\partial}{\partial t}\right\rangle=0,
$$

which gives $\frac{\partial g_{i j}}{\partial t}=0$ and concludes the proof. 
For every coordinate system $(U, \varphi)$ such that (C.1) holds, the surfaces

$$
t \equiv \text { const. }
$$

are transversal to $Y$; it is clear that for every point we can choose such a coordinate system such that these surfaces are spacelike, ${ }^{4}$ i.e. such that the restriction of the Lorentzian metric $g$ on them is positive definite. Such a coordinate system is said to be adapted to $Y$.

It should be remarked that, differently from the situation studied in [6], [8] and [11], in our setting $t$ is not a globally defined time function.

Then, $\mathcal{M}$ can be written locally as a topological product $\Sigma \times] a, b[$, where $\Sigma$ is a spacelike hypersurface parametrized by coordinates $x_{1}, x_{2}, \ldots, x_{N-1}$, and $] a, b\left[\right.$ is an open interval of $\mathbb{R}$. In coordinates $\mathbf{x}=\left(x_{1}, \ldots, x_{N-1}\right)$ and $t \in] a, b[$, the metric $g$ is written as:

$$
g(\mathbf{x}, t)[(\xi, \tau),(\xi, \tau)]=\langle\xi, \xi\rangle_{0}+2\langle\delta(\mathbf{x}), \xi\rangle_{0} \tau-\beta(\mathbf{x}) \tau^{2},
$$

where $(\xi, \tau) \in T_{x} \Sigma \times \mathbb{R},\langle\cdot, \cdot\rangle_{0}$ is the (positive definite) restriction of $g$ to $\Sigma$, $\delta$ is a smooth vector field on $\Sigma$ given by:

$$
\delta(\mathbf{x})=G_{0}(\mathbf{x})^{-1} \cdot \bar{\delta}(\mathbf{x}),
$$

where

$$
\bar{\delta}(\mathbf{x})=\sum_{i=1}^{N-1} g_{i N}(\mathbf{x}) \frac{\partial}{\partial x_{i}},
$$

$G_{0}(\mathbf{x})$ is the $(N-1) \times(N-1)$ matrix:

$$
G_{0}(\mathbf{x})=\left(g_{i j}\right)_{i, j=1}^{N-1},
$$

and $\beta$ is the smooth scalar field on $\Sigma$ given by:

$$
\beta(\mathbf{x})=-\langle Y, Y\rangle>0 .
$$

Using the definition (1.1) of $g_{(\mathrm{R})}$ it is easily computed:

$$
g_{(\mathrm{R})}[(\xi, \tau),(\xi, \tau)]=\langle\xi, \xi\rangle_{0}+\frac{2}{\beta(\mathbf{x})}\langle\delta(\mathbf{x}), \xi\rangle_{0}^{2}-2\langle\delta(\mathbf{x}), \xi\rangle_{0} \tau+\beta(\mathbf{x}) \tau^{2} ;
$$

\footnotetext{
${ }^{4}$ for instance, one can take an arbitrary coordinate system $\left(U_{z}, \varphi\right)$ such that (C.1) holds, choose a smooth function $S\left(x_{1}, x_{2}, \ldots, x_{N-1}\right)$ such that the graph $x_{N}=S\left(x_{1}, \ldots, x_{N-1}\right)$ passes through $z$ and is a spacelike hypersurface $\Sigma$ in $\mathcal{M}$, then foliate a neighborhood of $z$ using $\Sigma$ and the flow of $Y$.
} 
moreover, for an absolutely continuous curve $z(s)=(\mathbf{x}(s), t(s))$, the conservation law $\langle\dot{z}(s), Y(z(s))\rangle=C_{z}$ is given by:

$$
\langle\dot{z}(s), Y(z(s))\rangle=\langle\delta(\mathbf{x}(s)), \dot{\mathbf{x}}(s)\rangle_{0}-\beta(\mathbf{x}(s)) \dot{t}(s) \equiv C_{z} .
$$

Observe also that, in the metric (C.3), the gradient $\nabla t$ of the coordinate function $t$ is given by:

$$
\nabla t=\frac{1}{\beta+\langle\delta, \delta\rangle}(\delta,-1) .
$$

A quick computation gives:

$$
\langle\nabla t, \nabla t\rangle=-(\beta+\langle\delta, \delta\rangle)^{-1}<0,
$$

which says that $t$ is a local time function; moreover

$$
\langle\nabla t, Y\rangle \equiv 1
$$

\section{References.}

[1] F. Antonacci and P. Piccione, An Intrinsic Approach to a LjusternikSchnirelman Theory for Light Rays on Lorentzian Manifolds, to appear on Integral and Differential Equation, 1996.

[2] J. K. Beem, Conformal Changes and Geodesic Completeness, Comm. Math. Phys. 49 (1976), 179-186.

[3] J. K. Beem and H. Buseman, Axiom for Indefinite Metrics, Cir. Mat. Palermo 15 (1966), 223-246.

[4] J. K. Beem, P. E. Ehrlich and K. L. Easly, Global Lorentzian Geometry, Marcel Dekker, Inc., New York and Basel, 1996.

[5] J. K. Beem and P. E. Parker, Pseudoconvexity and Geodesic Connectedness, Ann. Mat. Pura e Applicata 155 (1989), 137-142.

[6] V. Benci, D. Fortunato and F. Giannoni, On the Existence of Infinitely Many Geodesics on Space-Time Manifolds, Ann. Inst. Henri Poincaré, Analyse Nonlinéaire, 8 (1991), 79-102.

[7] E. Fadell and S. Husseini, Category of Loop Spaces of Open Subsets in Euclidean Spaces, Nonlinear Analysis: T.M.A. 17 (1991), 1153-1161. 
[8] F. Giannoni and A. Masiello, On the Existence of Geodesics on Stationary Lorentz Manifolds with Convex Boundary, J. Funct. An. 101 (1991).

[9] S. W. Hawking and G. F. Ellis, The Large Scale Structure of Space-Time, Cambridge Univ. Press, London, New York, 1973.

[10] S. Lang, Differential Manifolds, Springer-Verlag, Berlin, 1985.

[11] A. Masiello, Variational Methods in Lorentzian Geometry, Pitman Research Notes in Mathematics Series 59, 1994.

[12] J. Mawhin and M. Willem, Critical Point Theory and Hamiltonian Systems, Springer-Verlag, Berlin, 1989.

[13] B. O'Neill, Semi-Riemannian Geometry with Applications to Relativity, Academic Press, New York, 1983.

[14] R. Palais, Morse Theory on Hilbert Manifolds, Topology 2 (1963), 299-340.

[15] A. Romero and M. Sánchez, New Properties and Examples of Incomplete Lorentzian Tori, J. Math. Phys. 34, 4 (1994), 1992-1997.

[16] A. Romero and M. Sánchez, Completeness of Compact Lorentz Manifolds Admitting a Timelike Conformal Killing Vector Field, Proc. AMS 123, 9 (1995), 2831-2833.

RECEIVEd February 21, 1997.

DipartimENTO Di ENERGETICA

FACOLTÁ DI INGEGNERIA

UNIVERSITÁ DELL'AQUILA

ITALY

AND

Departamento de Matemática

Instituto de Matemática e Estatística

Universidade de São Paulo

BRAZIL 